\title{
MAGNETIC FIELD AMPLIFICATION AND EVOLUTION IN TURBULENT COLLISIONLESS MAGNETOHYDRODYNAMICS: AN APPLICATION TO THE INTRACLUSTER MEDIUM
}

\author{
R. Santos-Lima ${ }^{1}$, E. M. De Gouveia Dal Pino ${ }^{1}$, G. Kowal ${ }^{1,2}$, \\ D. Falceta-GonÇAlves ${ }^{2,3}$, A. LAZARIAN ${ }^{4}$, AND M. S. NAKWACKI ${ }^{5,6}$ \\ ${ }^{1}$ Instituto de Astronomia, Geofísica e Ciências Atmosféricas, Universidade de São Paulo, R. do Matão, 1226, São Paulo, SP 05508-090, Brazil \\ ${ }^{2}$ Escola de Artes, Ciências e Humanidades, Universidade de São Paulo, Rua Arlindo Bettio, 1000, São Paulo, SP 03828-000, Brazil \\ ${ }^{3}$ SUPA, School of Physics \& Astronomy, University of St Andrews, North Haugh, St Andrews, Fife KY16 9SS, UK \\ ${ }^{4}$ Department of Astronomy, University of Wisconsin, Madison, WI 53706, USA \\ ${ }^{5}$ Instituto de Astronomía y Física del Espacio (IAFE), CONICET, Argentina \\ ${ }^{6}$ FCEN, University of Buenos Aires, Argentina \\ Received 2013 May 21; accepted 2013 November 20; published 2014 January 13
}

\begin{abstract}
The amplification of magnetic fields (MFs) in the intracluster medium (ICM) is attributed to turbulent dynamo (TD) action, which is generally derived in the collisional-MHD framework. However, this assumption is poorly justified a priori, since in the ICM the ion mean free path between collisions is of the order of the dynamical scales, thus requiring a collisionless MHD description. The present study uses an anisotropic plasma pressure that brings the plasma within a parametric space where collisionless instabilities take place. In this model, a relaxation term of the pressure anisotropy simulates the feedback of the mirror and firehose instabilities, in consistency with empirical studies. Our three-dimensional numerical simulations of forced transonic turbulence, aiming the modeling of the turbulent ICM, were performed for different initial values of the MF intensity and different relaxation rates of the pressure anisotropy. We found that in the high- $\beta$ plasma regime corresponding to the ICM conditions, a fast anisotropy relaxation rate gives results that are similar to the collisional-MHD model, as far as the statistical properties of the turbulence are concerned. Also, the TD amplification of seed MFs was found to be similar to the collisional-MHD model. The simulations that do not employ the anisotropy relaxation deviate significantly from the collisional-MHD results and show more power at the small-scale fluctuations of both density and velocity as a result of the action of the instabilities. For these simulations, the large-scale fluctuations in the MF are mostly suppressed and the TD fails in amplifying seed MFs.
\end{abstract}

Key words: galaxies: clusters: intracluster medium - magnetic fields - magnetohydrodynamics (MHD) turbulence

Online-only material: color figures

\section{INTRODUCTION}

Clusters of galaxies constitute the largest structures of the universe, and most of their baryonic mass is in the hot X-ray emitting gas that fills the intracluster medium (ICM). There, densities and temperatures are typically $10^{-2}-10^{-3}$ particles $/ \mathrm{cm}^{-3}$ (in the central regions) and $10^{7}-10^{8} \mathrm{~K}$, respectively (Laganá et al. 2008). Measurements of synchrotron emission and Faraday rotation of polarized radiation support the existence of magnetic fields in the ICM with inferred intensities of $\sim 1-10 \mu \mathrm{G}$ (Govoni \& Feretti 2004; Enßlin et al. 2005). At the same time, the ICM is expected to be turbulent due to the existence of numerous sources of turbulence there, the most energetic of which originate in mergers of galactic sub-clusters (see, e.g., Lazarian 2006a; Durret \& Lima Neto 2008; Govoni \& Feretti 2004; Enßlin et al. 2005).

The large-scale dynamics of the magnetized plasma in the ICM, commonly described by the magnetohydrodynamic (MHD) theory, links the evolution of the observed magnetic fields and the bulk motions of the gas. In this context, one of the most important outcomes from the MHD approximation is the ability of a driven turbulent flow to amplify the magnetic fields until close equipartition between kinetic and magnetic energies (Schekochihin et al. 2004). That is, once a seed magnetic field is present, turbulence will stretch and fold the field lines until the magnetic forces become dynamically important. Recent collisional MHD studies (Cho et al. 2009; Beresnyak 2012a; see also Brandenburg et al. 2012; de Gouveia Dal Pino et al. 2013 for reviews) show an efficient magnetic field amplification independent of the value of the initial seed field. This turbulent dynamo transfers about $6 \%$ of the energy flux of hydrodynamic cascade into magnetic energy. As the magnetic field enters into equipartition with hydrodynamic motions, the magnetic fields get correlation lengths of the order of the largest scales of the turbulence. While the origin of seed fields is still a matter of discussion, it little depends on the original value of the seed field, and different processes, e.g., of Biermann battery, can provide the sufficient value of the initial field (see, e.g., Lazarian 1992; Grasso \& Rubinstein 2001; de Gouveia Dal Pino 2006, 2011). The above turbulent dynamo scenario can be sustaining the magnetic fields in the ICM (Brandenburg \& Subramanian 2005; Brandenburg et al. 2012; de Gouveia Dal Pino et al. 2013, and references therein). This picture is supported by MHD simulations of galaxy mergers, showing the amplification of the magnetic field in the intergalactic medium (Kotarba et al. 2011).

However, one may wonder whether the results obtained within an MHD approximation can be applicable to the collisionless ICM, due to the small collision frequency of the protons compared to the frequencies of the turbulent motions and to the gyrofrequency of these particles around the field lines. The typical time and distance scales involved are: (1) for the injection scales of the turbulence: $\tau_{\text {turb }}=l_{\text {turb }} / v_{\text {turb }} \sim 100 \mathrm{Myr}$ considering $l_{\text {turb }} \sim 500 \mathrm{kpc}$ and $v_{\text {turb }} \sim 10^{3} \mathrm{~km} \mathrm{~s}^{-1}$ (e.g., 
Lazarian 2006a); (2) for the proton-proton (electron-electron) collision: $\tau_{\mathrm{pp}} \sim 30 \mathrm{Myr}\left(\tau_{\mathrm{ee}} \sim 1 \mathrm{Myr}\right)$ and the mean free path is $l_{\mathrm{pp}} \sim 30 \mathrm{kpc}\left(l_{\mathrm{ee}} \sim 1 \mathrm{kpc}\right)$; (3) for the proton (electrons) gyromotion: $\tau_{c p} \sim 10^{3} \mathrm{~s}\left(\tau_{\mathrm{ce}} \sim 1 \mathrm{~s}\right)$ and the Larmor radius $l_{\mathrm{ci}} \sim 10^{5} \mathrm{~km}\left(l_{\mathrm{ce}} \sim 10^{3} \mathrm{~km}\right)$. This makes the proton-proton collision rates negligible, enabling the thermalization of the energy of their motions in the different directions, if we consider only binary collisions. As a consequence, the particle velocity distributions parallel and perpendicular (gyromotions) to the magnetic field lines are decoupled (see Schekochihin et al. 2005; Kulsrud 2005).

The collisionless plasmas can be very different from its collisional counterpart. The unavoidable occurrence of temperature anisotropy is known from kinetic theory to trigger electromagnetic instabilities (see, for instance, Kulsrud 1983). These electromagnetic fluctuations, in turn, redistribute the pitch angles of the particles, decreasing the temperature anisotropy (Gary 1993; see also an alternative view in Schekochihin et al. 2010; Rosin et al. 2011; see Section 2). On the other hand, a fluid-like model is desirable for studying the large-scale plasma phenomena in the ICM, as well as the evolution of turbulence and magnetic fields there.

Fortunately, it is possible to describe many properties of collisionless plasma still using MHD-type models if additional constraints are applied. The simplest collisionless MHD-type approximation is the CGL-MHD model (Chew et al. 1956). A modified CGL-MHD model taking into account the anisotropy constraints due to kinetic instabilities has been used for modeling the solar wind and magnetosphere in numerical simulations (Denton et al. 1994; Pudovkin et al. 1999; Samsonov \& Pudovkin 2000; Samsonov et al. 2001, 2007; Meng et al. 2012a, 2012b; see also Chandran et al. 2011 where a higher order fluid model is used).

There has been previous important work on modeling collisionless and weakly collisional plasma within the MHD-type approach. For instance, Kunz et al. (2011) have proposed a semiphenomenological model of a thermally stable mechanism for heating the central regions of cold-core clusters of galaxies that is able to counterbalance the thermal emission losses, therefore preventing the nonobserved cooling flows. This heating is originated from thermalization of the turbulent energy by the viscous parallel heating (due to the Braginskii viscosity). They argue that the turbulence has an effective "impedance" that allows it to absorb only part of the power available from the stirring. This part corresponds to the amount that can be locally dissipated by viscosity without triggering micro-instabilities. One key hypothesis in their model is that the relaxation of the temperature anisotropy due to the kinetic instabilities is provided by the microscopic rearrangement of the magnetic field fluctuations, instead of the enhancement of the pitch-angle scattering of the particles - the effective collision rate remains constant (see details in Kunz et al. 2011).

In the context of rotating plasmas, Sharma et al. (2006) used collisionless MHD-type simulations to study the magnetorotational instability (MRI) in accretion disks around black holes. They found that the transport of angular momentum is enhanced by the stress originated from the anisotropic pressure.

More recently, Kowal et al. (2011b) performed a study of the turbulence statistics in a collisionless MHD-type model within a double-isothermal approximation (i.e., with the temperatures parallel and perpendicular to the local magnetic field assumed to be constant). Employing three-dimensional (3D) simulations for a set of parameters, they demonstrated how the presence of the instabilities driven by temperature anisotropy change the structure of the dynamical variables, i.e., density, magnetic field, and velocity. They reported substantial differences for the ordinary MHD and their collisionless MHD-type simulations of turbulence, for instance, substantial growth of turbulent energy at small scales.

At the same time, one may wonder whether the particles in collisionless fluids indeed behave similarly to their description in the MHD-type simulations above. For instance, Lazarian \& Beresnyak (2006) considered a fluid of magnetized cosmic rays and showed that instabilities in this fluid, such as the gyroresonance instability (see Gary 1993), decrease substantially the effective mean free path of otherwise collisionless particles. They also discussed the application of the same approach to collisionless plasmas (see also Schekochihin \& Cowley 2006). This approach was further developed in Yan \& Lazarian (2011), where mean free paths of particles were calculated using fully kinetic calculations. In other words, although the collisions of the particles between each other are unimportant, they cannot stream freely. Instead, they interact with the perturbations of the magnetic fields that are created by collective effects in the plasma. The very instabilities that were observed in numerical simulations (see Kowal et al. 2011b) provide feedback on the plasma particles, decreasing their mean free path. ${ }^{7}$

This approach stating that the actual mean free path of the particles is not, in general, determined by Spitzer cross sections of particles, but is due to complex plasma feedback, has been adopted in a number of works already, as mentioned above, particularly in the solar wind and magnetosphere context (Samsonov et al. 2001, 2007; Meng et al. 2012a, 2012b; Chandran et al. 2011). Also, Brunetti \& Lazarian (2011) calculated the efficiency of turbulent acceleration of cosmic rays in clusters of galaxies assuming that the ICM plasma has small effective mean free paths due to the effects discussed above. This, in agreement with observations, increased the efficiency of the acceleration due to the suppression of the collisionless damping of the compressible turbulence. In situ measurements of solar wind plasmas and other empirical plasma data also agree with the conclusion that collisionless plasmas do experience a high rate of collisions after all, but these collisions are not due to Coulomb interactions, but to particles interacting with the modes induced through instabilities. This also motivates us to explore how a plasma similar to that observed in solar wind would behave. ${ }^{8}$

The present work extends the previous study of Kowal et al. (2011b) and investigates the behavior of the turbulence and the amplification of the magnetic fields in the ICM, employing a collisionless MHD model with pressure anisotropy that is constrained by the feedback arising from the kinetic instabilities. For this goal, we perform 3D numerical simulations of forced turbulence in the presence of initial magnetic fields with different intensities. In Section 2, we discuss briefly the kinetic instabilities due to pressure anisotropy and their feedback on the plasma, which leads to non-linear saturation. In Section 3, we describe the one-fluid model setup for the collisionless plasma employed in this work. In Section 4, we describe our numerical

\footnotetext{
7 This is a sort of self-regulation in collisionless plasmas, when instabilities constrain their own growth through decreasing the effective mean free paths. 8 In terms of the simulations, one may expect that reducing the mean free path of the particles should make a collisional MHD approach applicable to describe such environments, as in the presence of instabilities one can expect rather efficient scattering.
} 
experiments and results, followed by discussions in Section 5. In Section 6, we summarize our conclusions.

\section{MHD MODEL FOR A COLLISIONLESS PLASMA}

Measurements from weakly collisional plasmas, as those in the solar wind and the Earth's magnetosphere, have demonstrated that the kinetic instabilities driven by pressure anisotropy are able to induce the pitch-angle scattering of plasma particles, thus decreasing the resulting anisotropy. This scattering can be calculated from first principles (e.g., Lazarian \& Beresnyak 2006; Yan \& Lazarian 2011), but the corresponding calculations are rather complicated if the feedback of a few instabilities should be calculated. Therefore, in this paper we adopt an empirical approach based on the measurements above. Besides all the available in situ cosmic plasma observations (Marsch 2006 and references therein), further motivation for the choice of our relaxation model is based on the fact that, regardless of the differences in collisionless plasma regimes and the anisotropy instabilities, analytical models (Hall 1979, 1980, 1981), quasi-linear calculations (Yoon \& Seough 2012; Seough \& Yoon 2012), particle-in-cell (PIC) simulations (Tajima et al. 1977; Tanaka 1993; Gary et al. 1997, 1998, 2000; Le et al. 2010; Nishimura et al. 2002; Qu et al. 2008; Riquelme et al. 2012), and laboratory experiments (Keiter 1999) evidence the existence of saturation of the temperature anisotropy at some level, originated from the microscopic instabilities. In particular, constraints on the anisotropy due to the mirror and firehose instabilities have been clearly detected from solar wind protons (see, for example, Hellinger et al. 2006; Bale et al. 2009) and $\alpha$-particles (Maruca et al. 2012).

In order to describe the evolution of turbulence in the collisionless plasma of the ICM, we employ the one-fluid CGL-MHD model, which is modified to take into account the anisotropy relaxation due to the feedback of kinetic instabilities. In the next paragraphs of this section we describe our assumptions and provide further justification for them (for further discussion see also Section 5).

We can write the equations of the model in the following conservative form:

$$
\begin{gathered}
\frac{\partial}{\partial t}\left[\begin{array}{c}
\rho \\
\rho \mathbf{u} \\
\mathbf{B} \\
e \\
A\left(\rho^{3} / B^{3}\right)
\end{array}\right]+\nabla \cdot\left[\begin{array}{c}
\rho \mathbf{u} \\
\rho \mathbf{u u}+\Pi_{P}+\Pi_{B} \\
\mathbf{u B}-\mathbf{B u} \\
e \mathbf{u}+\mathbf{u} \cdot\left(\Pi_{P}+\Pi_{B}\right) \\
A\left(\rho^{3} / B^{3}\right) \mathbf{u}
\end{array}\right] \\
=\left[\begin{array}{c}
0 \\
\mathbf{f} \\
0 \\
\mathbf{f} \cdot \mathbf{v}+\dot{w} \\
\dot{A}_{S}\left(\rho^{3} / B^{3}\right)
\end{array}\right],
\end{gathered}
$$

where $\rho, \mathbf{u}, \mathbf{B}$, and $p_{\perp, \|}$ are the macroscopic variables density, velocity, magnetic field, and thermal pressures perpendicular/ parallel to the local magnetic field, respectively; $e=\left(p_{\perp}+\right.$ $\left.p_{\|} / 2+\rho u^{2} / 2+B^{2} / 8 \pi\right)$ is total energy density, and $A=p_{\perp} / p_{\|}$ is the anisotropy in the pressure. $\Pi_{P}$ and $\Pi_{B}$ are the gyrotropic pressure and the magnetic stress tensors, respectively, defined by

$$
\Pi_{P}=p_{\perp} \mathbf{I}+\left(p_{\|}-p_{\perp}\right) \mathbf{b b}
$$

and

$$
\Pi_{B}=\left(B^{2} / 8 \pi\right) \mathbf{I}-\mathbf{B B} / 4 \pi,
$$

where $\mathbf{I}$ is the unitary dyadic tensor and $\mathbf{b}=\mathbf{B} / B$. In the source terms, $\mathbf{f}$ represents an external bulk force responsible for driving the turbulence (see details in Section 3.1), $\dot{w}$ gives the rate of change of the internal energy $w=\left(p_{\perp}+p_{\|} / 2\right)$ of the gas due to heat conduction and radiative cooling, and $\dot{A}_{S}$ gives the rate of change of $A$ due to microphysical processes (see Section 2.2). Before specifying the details of these source terms, we briefly present the dispersion relation of the waves and instabilities in a CGL-MHD homogeneous system.

\subsection{CGL-MHD Waves and Instabilities}

In the absence of the source terms in Equation (1), we recover the standard CGL-MHD model. In this model, first obtained by Chew et al. (1956), the evolution of the pressure components is expressed by

$$
\frac{d}{d t}\left(\frac{p_{\perp}}{\rho B}\right)=0, \quad \frac{d}{d t}\left(\frac{p_{\|} B^{2}}{\rho^{3}}\right)=0,
$$

which is also called the double-adiabatic law.

Linear perturbation analysis of the CGL-MHD equations reveals three waves, analogous to the Alfvén, slow, and fast magnetosonic MHD waves. These waves, however, can have imaginary frequencies for sufficiently high degrees of the pressure anisotropy. The corresponding dispersion relations can be found in Kulsrud (1983). For convenience, we reproduce here these relations (using the same notation as in Hau \& Wang 2007):

$$
\begin{gathered}
\left(\frac{\omega}{k}\right)_{a}^{2}=\left(\frac{B^{2}}{4 \pi \rho}+\frac{p_{\perp}}{\rho}-\frac{p_{\|}}{\rho}\right) \cos ^{2} \theta, \\
\left(\frac{\omega}{k}\right)_{f, s}^{2}=\frac{b \pm \sqrt{b^{2}-4 c}}{2},
\end{gathered}
$$

where $\cos \theta=\mathbf{k} \cdot \mathbf{B} / B$ ( $\mathbf{k}$ being the wavevector of the perturbation) and

$$
\begin{gathered}
b=\frac{B^{2}}{4 \pi \rho}+\frac{2 p_{\perp}}{\rho}+\left(\frac{2 p_{\|}}{\rho}-\frac{p_{\perp}}{\rho}\right) \cos ^{2} \theta \\
c=-\cos ^{2} \theta\left[\left(\frac{3 p_{\|}}{\rho}\right)^{2} \cos ^{2} \theta-\frac{3 p_{\|}}{\rho} b+\left(\frac{p_{\perp}}{\rho}\right)^{2} \sin ^{2} \theta\right] .
\end{gathered}
$$

The dispersion relation for the transverse (Alfvén) mode $(\omega / k)_{a}^{2}$ coincides with that obtained from the kinetic theory (in the limit when the Larmor radius goes to zero) and does not change when heat conduction is added to the system (see Kulsrud 1983); the criterion for the instability (named firehose instability), in terms of $A=p_{\perp} / p_{\|}$and $\beta_{\|}=p_{\|} /\left(B^{2} / 8 \pi\right)$, is in this case

$$
A<1-2 \beta_{\|}^{-1} \text {. }
$$

However, for the compressible modes $(\omega / k)_{f, s}^{2}$ (which include the mirror unstable modes), the linear dispersion relation of the CGL-MHD equations is known to deviate from the kinetic theory. The mirror instability criterion is

$$
A / 6>1+\beta_{\perp}^{-1},
$$

while the one derived from the kinetic theory is

$$
A>1+\beta_{\perp}^{-1},
$$

where $\beta_{\perp}=p_{\perp} /\left(B^{2} / 8 \pi\right)$ in the last two expressions. 
Taking into account the finite Larmor radius effects, Meng et al. (2012a) (see also Hall 1979, 1980, 1981) give the following expressions for the maximum growth rate $\gamma_{\max }$ (normalized by the ion gyrofrequency $\Omega_{i}$ ) of the firehose and kinetic mirror instabilities:

$$
\frac{\gamma_{\max }}{\Omega_{i}}= \begin{cases}\frac{1}{2} \frac{\left(1-A-2 \beta_{\|}^{-1}\right)}{\sqrt{A-1 / 4}} & \text { (firehose) } \\ \frac{4 \sqrt{2}}{3 \sqrt{5}} \sqrt{A-1-\beta_{\perp}^{-1}} & \text { (mirror) },\end{cases}
$$

which are achieved for $k^{-1} \sim l_{\text {ci }}$, the ion Larmor radius. These expressions are valid for the case of $|A-1| \ll 1$ and $\beta_{\|, \perp} \gg 1$.

\subsection{Pressure Anisotropy Relaxation}

Following previous works (Denton et al. 1994; see also Pudovkin et al. 1999; Samsonov \& Pudovkin 2000; Samsonov et al. 2001; Meng et al. 2012a), whenever the plasma satisfies the firehose (Equation (7)) or kinetic mirror instability criteria (Equation (9)), we impose the following pressure anisotropy relaxation condition:

$$
\left(\frac{\partial p_{\perp}}{\partial t}\right)_{S}=-\frac{1}{2}\left(\frac{\partial p_{\|}}{\partial t}\right)_{S}=-v_{S}\left(p_{\perp}-p_{\perp}^{*}\right),
$$

where $p_{\perp}^{*}$ is the value of $p_{\perp}$ for the marginally stable state (which is obtained from the equality in Equations (7) and (9) for each instability and with the conservation of the thermal energy $w$ ). The way by which Equation (11) is related to the rate of change of the anisotropy $\dot{A}_{S}$ of Equation (1) is discussed in Section 3.1.

It is not clear yet how the saturation and isotropization timescales are related to the local physical parameters. Some authors claim that the values of $v_{S}$ are of the order of the maximum growth rate of each instability $\gamma_{\max }$, which in turn is a fraction of the ion Larmor frequency $\gamma_{\max } / \Omega_{i} \sim 10^{-2}-10^{-1}$ (see Gary et al. 1997, 1998, 2000). In the ICM, the frequency $\Omega_{i}$ is very large compared to the frequencies that we resolve numerically. This implies that $v_{S} \rightarrow \infty$ would be a good approximation, or in other words, the relaxation to the marginal values would be instantaneous (which is similar to the "hardwalls" employed in Sharma et al. 2006). However, it is not clear yet whether the extreme low density and weak magnetic fields of the ICM would result in isotropization timescales as fast as these. Therefore, we have also tested, for comparison, finite values for $v_{S}$ that are $\ll \Omega_{i}$ (see Section 3.3).

\subsection{Thermal Relaxation}

The CGL-MHD model neglects any heat conduction or radiative cooling mechanisms, which is not a realistic assumption for the ICM. Combining Equations (4), we find that

$$
w^{*}=\left[\left(\frac{B}{B_{0}}\right) A_{0}+\frac{1}{2}\left(\frac{B}{B_{0}}\right)^{-2}\left(\frac{\rho}{\rho_{0}}\right)^{2}\right] \frac{w_{0}^{*}}{\left(A_{0}+1 / 2\right)},
$$

where $w^{*}=\left(p_{\perp}+p_{\|} / 2\right) / \rho$, and the subscripts 0 refer to the initial values in the Lagrangian fluid volume.

In the statistically steady state of the turbulence, the constant turbulent dissipation power leads to a secular increasing of the temperature of the gas, which can lead to heat conduction and radiative losses. In order to deal with these effects in a simplified
Table 1

Parameters of the Simulated Models

\begin{tabular}{lccccccc}
\hline \hline Name & $v_{S}$ & $v_{\text {th }}$ & $V_{A 0}$ & $V_{S 0}$ & $\beta_{0}$ & $t_{f}$ & Resolution \\
\hline A1 & $\infty$ & 5 & 0.3 & 1 & 200 & 5 & $512^{3}$ \\
A2 & 0 & 5 & 0.3 & 1 & 200 & 5 & $512^{3}$ \\
A3 & $10^{1}$ & 5 & 0.3 & 1 & 200 & 5 & $512^{3}$ \\
A4 & $10^{2}$ & 5 & 0.3 & 1 & 200 & 5 & $512^{3}$ \\
A5 & $10^{3}$ & 5 & 0.3 & 1 & 200 & 5 & $512^{3}$ \\
A6 & $\infty$ & 0 & 0.3 & 1 & 200 & 5 & $512^{3}$ \\
A7 & $\infty$ & 0.5 & 0.3 & 1 & 200 & 5 & $512^{3}$ \\
A8 & $\infty$ & 50 & 0.3 & 1 & 200 & 5 & $512^{3}$ \\
Amhd & $\cdots$ & 5 & 0.3 & 1 & 200 & 5 & $512^{3}$ \\
\hline B1 & $\infty$ & 5 & 3.0 & 1 & 0.2 & 5 & $512^{3}$ \\
Bmhd & $\ldots$ & 5 & 3.0 & 1 & 0.2 & 5 & $512^{3}$ \\
\hline C1 & $\infty$ & 5 & $10^{-3}$ & 1 & $2 \times 10^{6}$ & 40 & $256^{3}$ \\
C2 & 0 & 5 & $10^{-3}$ & 1 & $2 \times 10^{6}$ & 40 & $256^{3}$ \\
C3 & 0 & 5 & $10^{-3}$ & 0.3 & $2 \times 10^{5}$ & 40 & $256^{3}$ \\
C4 & $10^{2}$ & 5 & $10^{-3}$ & 1 & $2 \times 10^{6}$ & 40 & $256^{3}$ \\
Cmhd & $\cdots$ & 5 & $10^{-3}$ & 1 & $2 \times 10^{6}$ & 40 & $256^{3}$ \\
\hline
\end{tabular}

way, we employ a term $\dot{w}$ that relaxes the specific internal energy $w^{*}$ to the initial value $w_{0}^{*}$ at a constant rate $v_{\text {th }}$ (see a similar approach in Brandenburg et al. 1995):

$$
\dot{w}=-v_{\text {th }}\left(w^{*}-w_{0}^{*}\right) \rho .
$$

Although simplistic, this approximation is useful for two reasons: (1) it allows the system to dissipate the turbulent power excess; and (2) it helps to relax the local values of $w^{*}$, which may become artificially high or low in the CGL-MHD formulation without constraints on the anisotropy growth (see discussion in Section 5.2).

\section{NUMERICAL METHODS AND SETUP}

\subsection{The Code}

Equations (1) were evolved in a 3D Cartesian box employing a modified version of the shock-capturing, second-order Godunov code (Kowal et al. 2011b). The numerical fluxes were calculated using the HLL Riemann solver, with the maximum characteristic speed evaluated from Equations (5) and (6). For the time integration we used the second-order Runge-Kutta method (RK2).

The induction equation was integrated in its "uncurled" form, in an equivalent way to the Constrained Transport method. In the simulations presented in this work, no explicit diffusion term was used, except for model A2 (see Table 1), where an ohmic dissipation term with a diffusivity $\eta \sim 10^{-4}$ (i.e., of the same order of the numerical diffusivity) was employed in order to prevent eventual negative values of the internal energy $w$. These can arise because the eventual diffusion of the magnetic energy, especially in the presence of very high spatial frequency instabilities, is not being explicitly taken into account in the energy equation. Nonetheless, numerical tests showed that the introduction of this diffusion term does not cause significant differences in the results for this model.

To prevent negative values of the anisotropy $A$ due to precision errors during the numerical integration, we used an equivalent logarithmic formulation of the last equation in (1), which in the absence of source terms is given by

$$
\frac{\partial}{\partial t}\left[\rho \ln \left(A \rho^{2} / B^{3}\right)\right]+\nabla \cdot\left[\rho \ln \left(A \rho^{2} / B^{3}\right) \mathbf{u}\right]=0 .
$$


The pressure anisotropy relaxation was applied after each sub-time step of the RK2 method, by transforming the conservative variables $e$ and $A$ into the primitive ones $p_{\perp}$ and $p_{\|}$, calculating their relaxed values through Equation (11) (using the same implicit method as in Meng et al. 2012b) $)^{9}$ and then reconstructing back the conservative variables.

The turbulence (represented by the source term $\mathbf{f}$ in Equation (1)) is driven by adding a solenoidal velocity field to the domain at the end of each time step. This velocity field is calculated in the Fourier space with a random (but solenoidal) distribution in directions and sharply centered in a chosen value $k_{\text {turb }}$ (being the injection scale $l_{\text {turb }}=L / k_{\text {turb }}$, where $L$ is the size of the cubic domain). The forcing is approximately delta correlated in time.

The time-step constraint based on the Courant stability criterion $\delta t_{C}$ is calculated taking into account both the real and the imaginary characteristic speeds of the linear modes (Equations (5) and (6)).

Another time-step constraint is considered due to the thermal relaxation (Equation (13)). At the end of each time step, we estimate the minimum characteristic time of the thermal relaxation $\delta t_{\text {th }}$ for the next time step as given by

$$
\delta t_{\mathrm{th}}=\min \left(\frac{w}{\dot{w}}\right)
$$

where $\dot{w}$ is the value calculated during the time step and the minimum value is computed over the whole domain.

The next time step is then taken as the minimum between $\epsilon_{C} \delta t_{C}$ and $\epsilon_{\mathrm{th}} \delta t_{\mathrm{th}}$ where, after performing several tests, we have chosen the factors $\epsilon_{C}=0.3$ and $\epsilon_{\mathrm{th}}=0.1$.

\subsection{Reference Units}

In the next sections, all the physical quantities are given in code units and can be easily converted in physical units using the reference physical quantities described below.

We arbitrarily choose three representative quantities from which all the other ones can be derived: a length scale $l_{*}$ (which is given by the computational box side), a density $\rho_{*}$ (given by the initial ambient density of the system), and a velocity $v_{*}$ (given by the initial sound speed in most of the models, but Model C3 for which the velocity unit is $0.3 v_{*}$; see Table 1 ). For instance, with such representative quantities the physical timescale is given by the time in code units multiplied by $l_{*} / v_{*}$; the physical energy density is obtained from the energy value in code units times $\rho_{*} v_{*}^{2}$, and so on. The magnetic field in code units is already divided by $\sqrt{4 \pi}$; thus, to obtain the magnetic field in physical units, one has to multiply the value in code units by $v_{*} \sqrt{4 \pi \rho_{*}}$.

\subsection{Initial Conditions and Parametric Choice}

Table 1 lists the simulated models and their initial parameters.

In Table $1, V_{A 0}$ is the Alfvén speed given by the initial intensity of the magnetic field directed along the $x$-axis. Initially, the gas pressure is isotropic for all the models with an isothermal sound speed $V_{S 0}$. The parameter $\beta_{0}$ is the initial ratio between the thermal pressure and the magnetic pressure.

\footnotetext{
9 In the case $v_{S}=\infty$ (see Table 1$)$, when the plasma is in the firehose (Equation (7)) or in the kinetic mirror instability (Equation (9)) regimes, this method simply replaces the pressures $p_{\perp}$ and $p_{\|}$by the values given by the corresponding marginal stability criterion (while ensuring conservation of the internal energy $w=p_{\perp}+p_{\|} / 2$ ).
}

Turbulence was driven considering the same setup in all the models of Table 1 . The injection scale is $l_{\text {turb }}=0.4$. The power of injected turbulence $\epsilon_{\text {turb }}$ is kept constant and equal to unity. After $t=1$ a fully turbulent flow develops in the system with an rms velocity $v_{\text {turb }}$ close to unity. This implies a turbulent turnover (or cascading) time $t_{\text {turb }} \approx 0.4$.

Models A, B, and C in Table 1 are collisionless MHD models with initial moderate, strong, and very small (seed) magnetic fields, respectively. For models $\mathrm{A}$ and $\mathrm{C}$ the injected turbulence is initially super-Alfvénic, while for models B it is sub-Alfvénic.

Amhd, Bmhd, and Cmhd correspond to collisional MHD models, i.e., have no anisotropy in pressure. The set of equations describing these models is identical to those in Equation (1), but dropping the equation for the evolution of the anisotropy $A$ and replacing the thermal energy by $w=3 p / 2$ (which corresponds to a polytropic gas index 5/3). Their corresponding dispersion relations are those from the usual collisional MHD approach (rather than Equations (5) and (6)).

To better explore the parameter space, we have also considered different values of the pressure anisotropy relaxation rate $v_{S}$. Models with $v_{S}=\infty$ (i.e., with instantaneous relaxation rate) represent conditions for which the relaxation time $\sim v_{S}^{-1}$ is much shorter than the minimum time step $\delta t_{\min }$ that our numerical simulations are able to solve $\left(\delta t_{\min } \sim 10^{-6}\right)$. Previous studies (Gary et al. 1997, 1998, 2000) suggest that the rate $v_{S}$ should be of the order of a few percent of $\Omega_{p}$, the proton gyrofrequency (see Section 5). If we consider typical physical conditions for the ICM, in order to convert the code units into physical units (see Section 3.2), we may take $l_{*}=100 \mathrm{kpc}, v_{*}=10^{8} \mathrm{~cm} \mathrm{~s}^{-1}$, and $\rho_{*}=10^{-27} \mathrm{~g} \mathrm{~cm}^{-3}$ as characteristic values for the length scale, dynamical velocity, and density of the ICM, respectively. This implies a characteristic timescale $t_{*} \sim 10^{15} \mathrm{~s}$, while for models A in Table 1, the proton Larmor period is $\tau_{c p} \sim 10^{3} \mathrm{~s}$. Using $v_{S} \sim 10^{-3} \tau_{c p}^{-1}$, we find $v_{S}^{-1} \sim 10^{-9} t_{*}$. Therefore, the models of Table 1 for which we assumed $v_{S}=\infty$ are very good approximations to the description of the direct effect of plasma instabilities at the large-scale turbulent motions within the ICM. For comparison, we have also run models with no anisotropy relaxation, or $v_{S}=0$, which thus behave like standard CGLmodels.

We notice that the turbulence in the ICM is expected to be trans- or even subsonic, and the plasma $\beta$ is expected to be high $(\beta \sim 200)$. Therefore, models A are possibly more representative of the typical conditions in the ICM.

In the following section we will start by describing the results for models $\mathrm{A}$ and $\mathrm{B}$, which have initial finite magnetic fields and therefore reach a nearly steady state turbulent regime relatively rapidly after the injection of turbulence. Then, we will describe models $\mathrm{C}$, which start with seed magnetic fields and therefore undergo a dynamo amplification of field due to the turbulence and take much longer to reach a nearly steady state.

\section{RESULTS}

Figure 1 depicts the density (top row) and the magnetic intensity (bottom row) distribution maps of the central slices for collisionless models with moderate initial magnetic fields A2 (left column), A1 (middle column), and Amhd (right column). All these models have $\beta_{0}=200$ and the same initial conditions, except for the anisotropy relaxation rate $v_{S}$.

In the A2 model there is no constraint on the growth of the pressure anisotropy $\left(v_{S}=0\right)$. In this case, the kinetic instabilities that develop due to the anisotropic pressure are 
A2: $\beta_{0}=200, \nu_{S}=0$

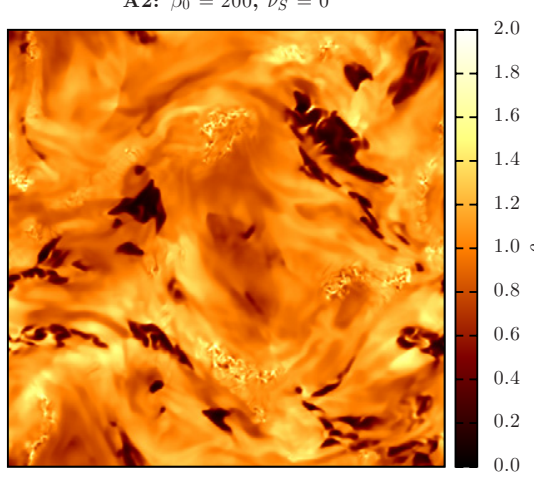

A2: $\beta_{0}=200, \nu_{S}=0$

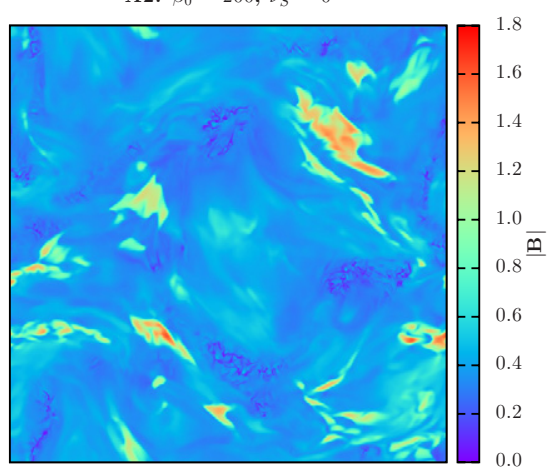

A1: $\beta_{0}=200, \nu_{S}=\infty$

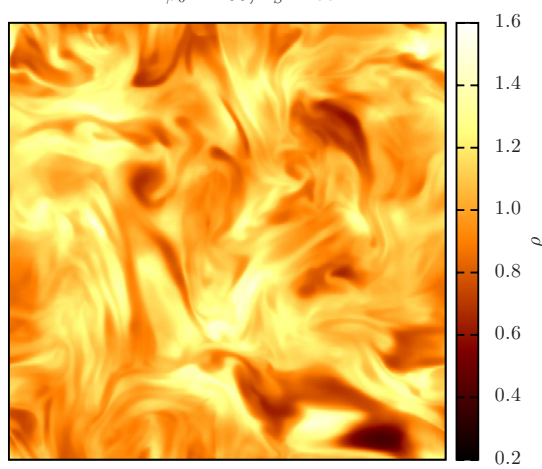

A1: $\beta_{0}=200, \nu_{S}=\infty$

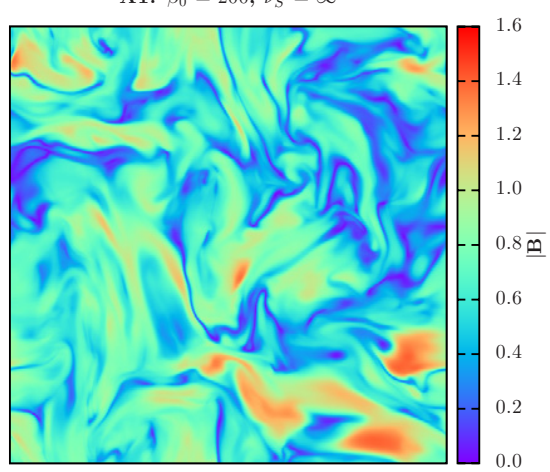

Amhd: $\beta_{0}=200$

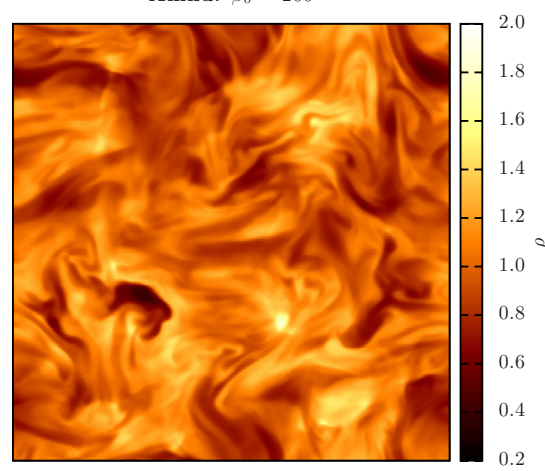

Amhd: $\beta_{0}=200$

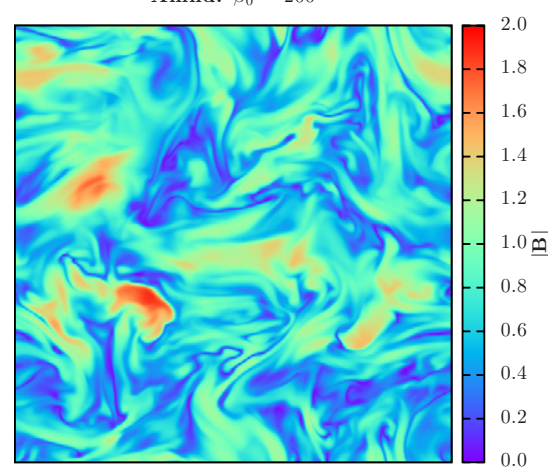

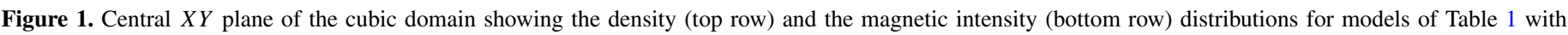

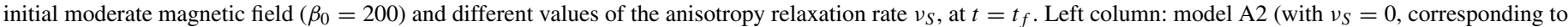

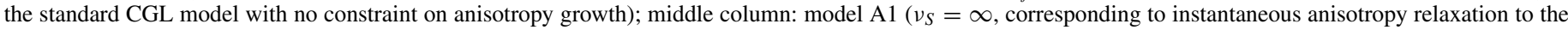

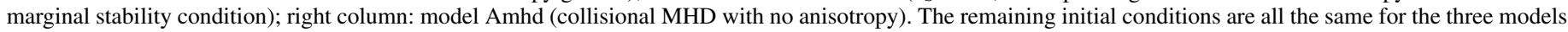
(see Table 1).

(A color version of this figure is available in the online journal.)

very strong at the smallest scales. This makes the density (and the magnetic field intensity) distribution in Figure 1 more "wrinkled" than in the standard (collisional) MHD case. On the other hand, in the A1 model where the isotropization of the thermal pressure due to the back-reaction of the same kinetic instabilities is allowed to occur above a threshold, the developed density (and magnetic field intensity) structures are larger than and more similar to those of the collisional MHD turbulent model Amhd.

In order to better quantify and understand the results evidenced by Figure 1 regarding the collisionless models without and with anisotropy growth constraints, in the next paragraphs of this section we will present a statistical analysis of the physical variables of these turbulent models after they reach a steady state.

For models A1 to Bmhd in Table 1, the statistical analyses were performed by averaging data from snapshots taken every $\Delta t=1$, from $t=2$ until the final time step $t_{f}$ indicated in Table 1. For the models with initial seed fields, $\mathrm{C} 1$ to $\mathrm{Cmhd}$, the statistical analysis considered snapshots from $t=\left(t_{f}-10\right)$ until $t_{f}$.

Averages and standard deviation of important physical quantities that will be discussed below are presented in Tables 2-4 (in the Appendix).

\subsection{The Role of the Anisotropy and Instabilities}

The injected turbulence produces shear and compression in the gas and in the magnetic field. Under the collisionless approximation, according to Equations (4) $A \propto B^{3} / \rho^{2}$; therefore, one should expect that compressions along the magnetic field lines, which keep $B$ constant but make $\rho$ increase, cause a decrease of $A$, while compressions or shear perpendicular to the magnetic field lines, which make $B$ increase but keep either $B / \rho$ or $\rho$ constant, cause an increase of $A$. Therefore, even starting with $A=1$, parcels of the gas with $A \neq 1$ will naturally develop. Inside these parcels, kinetic instabilities can be triggered, which in turn will inhibit the growth of the anisotropy.

Figure 2 presents the distribution of the anisotropy $A$ as a function of $\beta_{\|}$for the models with moderate initial magnetic field A1, A2, A3, A4, and A5 of Table 1. Model A2 ( $\left.v_{S}=0\right)$ has an A distribution that nearly follows a line with negative inclination in the log-log diagram. This is consistent with the derived $A$ dependence in the CGL models given by $A \propto(\rho / B) \beta_{\|}^{-1}$ (when the initial conditions are homogeneous; see Equations (4)). This model attains values of $A$ spanning several orders of magnitude (from $10^{-2}$ to $10^{3}$ ).

Model A1 $\left(v_{S}=\infty\right)$, on the other hand, keeps $A$ close to unity, varying by less than one order of magnitude.

Figure 2 also shows the distribution of $A$ for the A3, A4, and A5 models, which have bounded anisotropy with finite anisotropy relaxation rates $\nu_{S}$ (see Table 1). We see that in these cases, a fraction of the gas has $A$ values out of the stable zone. The model with smaller anisotropy relaxation rate (model A3) obviously presents a larger fraction of gas inside the unstable zones. We also note that the higher the value of $\beta_{\|}$, the larger the linear growth rate of the instabilities and the more gas is inside the unstable regions with $A<1$. This is consistent with the CGL trend for which $A \propto \beta_{\|}^{-1}$.

Bottom right panel of Figure 2 shows the distribution of $A$ versus $\beta_{\|}$for the model B1 with strong initial magnetic field 

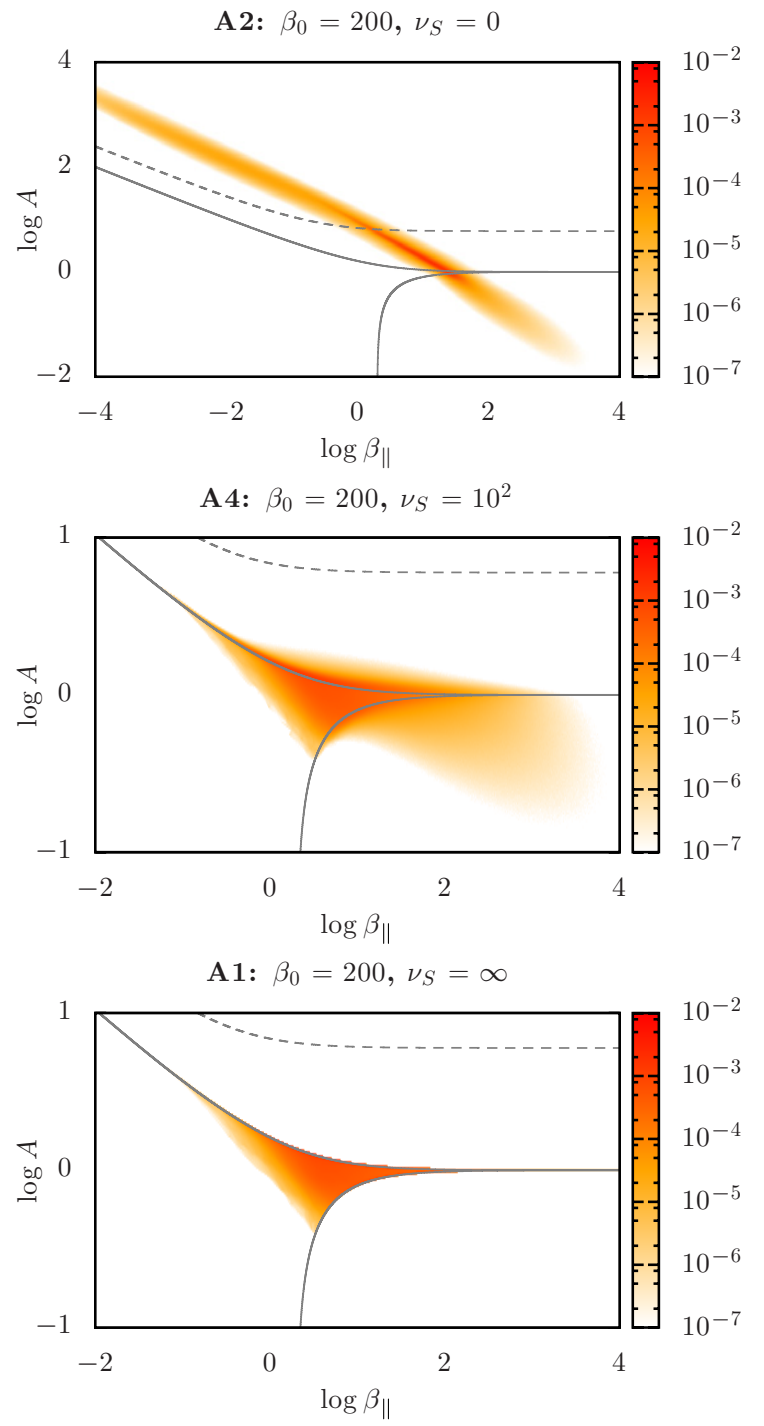
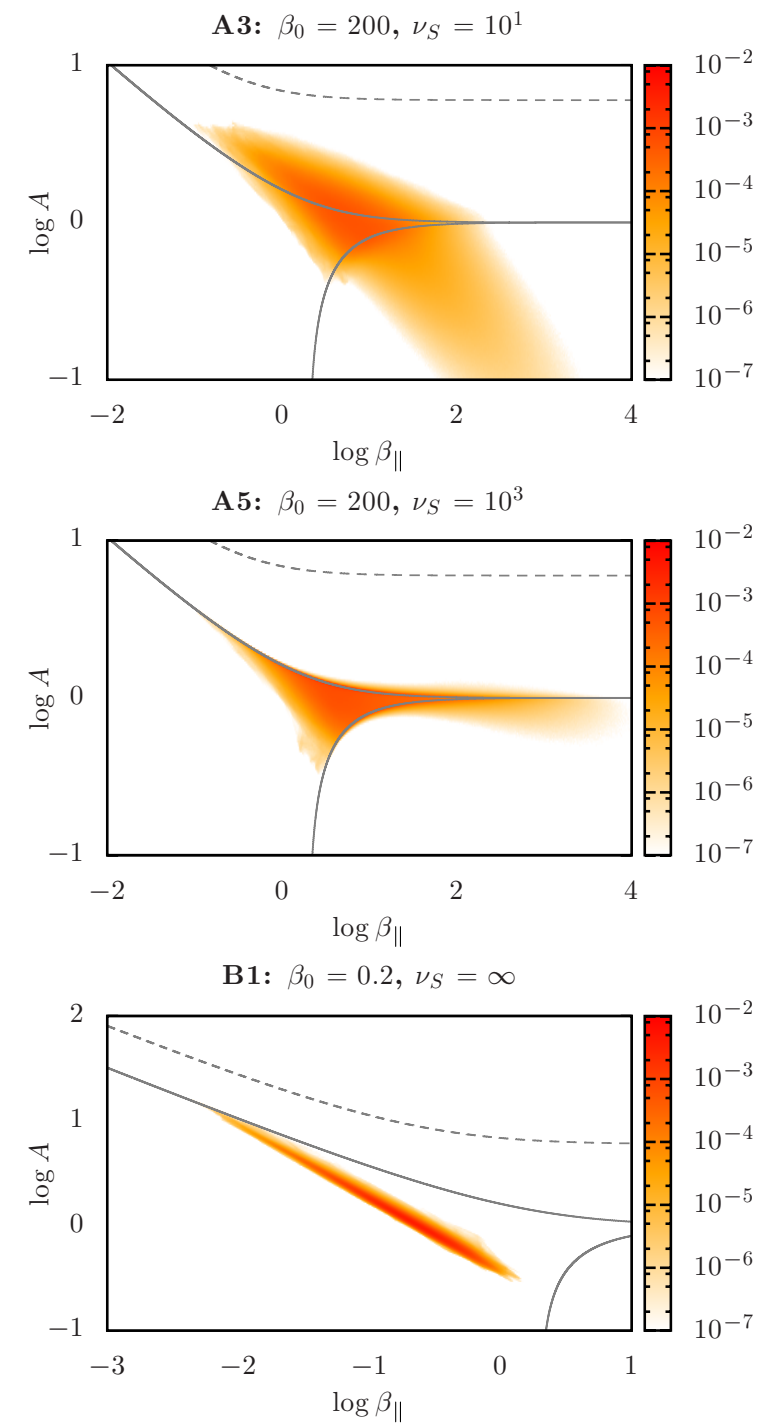

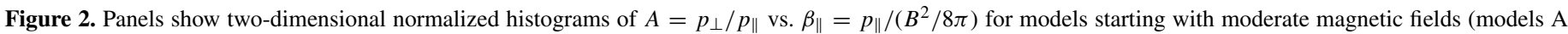

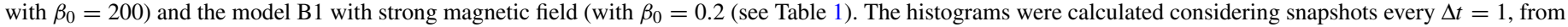

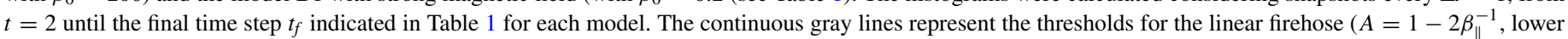

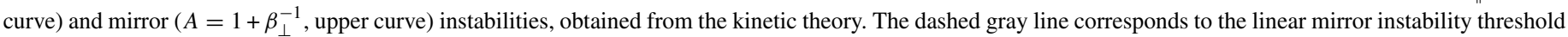
obtained from the CGL-MHD approximation $\left(A / 6=1+\beta_{\perp}^{-1}\right)$.

(A color version of this figure is available in the online journal.)

(small $\beta_{0}=0.2$ ). We see that, in this regime, model B1 has an $A$ distribution inside the stable zone.

The spatial anisotropy distribution is illustrated in Figure 3 in two-dimensional (2D) maps that depict central slices of $A$ in the $X Y$-plane at the final time step for models $\mathrm{A} 1, \mathrm{~A} 2$, $\mathrm{A} 3$, A4, A5, and B1. For the CGL model with moderate magnetic field $\left(\beta_{0}=200\right)$, model $\mathrm{A} 2$, the $A$ structures are thin and elongated. These small-scale structures probably arise from the fast fluctuations driven by the kinetic instabilities (see Figure 2). For the model with strong magnetic field (small $\beta_{0}$ ), model B1, the $A$ structures are smoother. They are originated by small-amplitude magnetic fluctuations (Alfvén waves) and also compression modes at the large scales. The map of model A1 also shows thin and elongated structures, but with lengths of the order of the turbulence scale.

As an illustration of the spatial distribution of the unstable gas, Figure 4 depicts maps of the maximum growth rate of both the firehose (left column) and the mirror (right column) instabilities given by Equations (10) for the models with moderate initial magnetic field $\left(\beta_{0}=200\right)$ and different anisotropy relaxation rates $v_{S} .{ }^{10}$ These maximum growth rates are normalized by the initial ion gyrofrequency $\Omega_{i 0}$ and occur for modes with wavelengths of the order of the ion Larmor radius. The first thing to note is that the mirror unstable regions have a larger volume filling factor than the firehose unstable regions for all the models in Figure 4. This is because the regions where the magnetic field is amplified have a large perpendicular pressure, and this happens on most of the turbulent volume. Regions with an excess of parallel pressure arise when the magnetic intensity decays, like in regions with magnetic field reversals

\footnotetext{
10 We note that because Equations (10) have a validity limit as described in Section 2.1, we have corrected the growth rates to $\gamma_{\max } / \Omega_{i}=1$ when outside of the validity range. This limit is well justified by solutions of the dispersion relation obtained from the linearization of the Vlasov-Maxwell equation by Gary (1993; see Chapter 7).
} 
A2: $\beta_{0}=200, \nu_{S}=0$

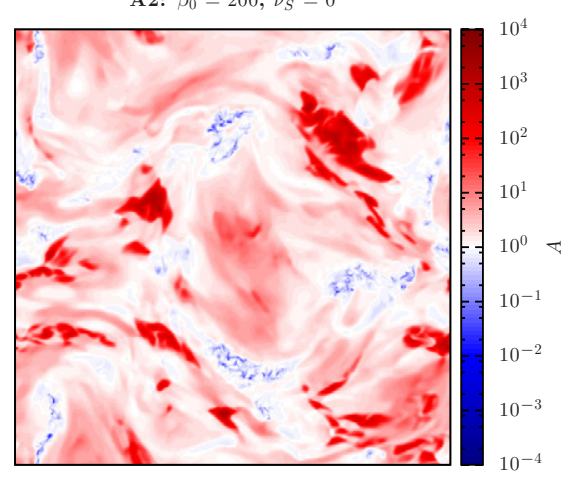

A5: $\beta_{0}=200, \nu_{S}=10^{3}$

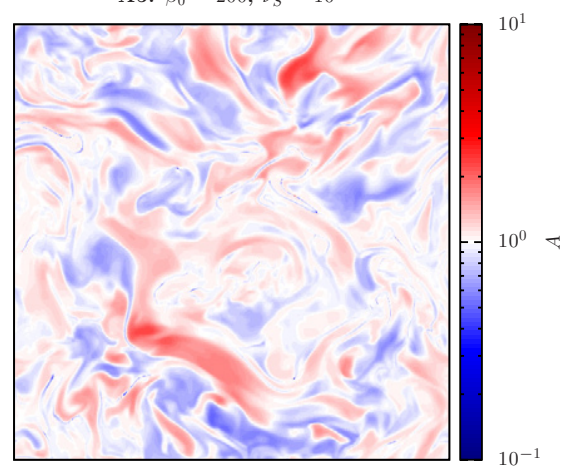

A3: $\beta_{0}=200, \nu_{S}=10^{1}$

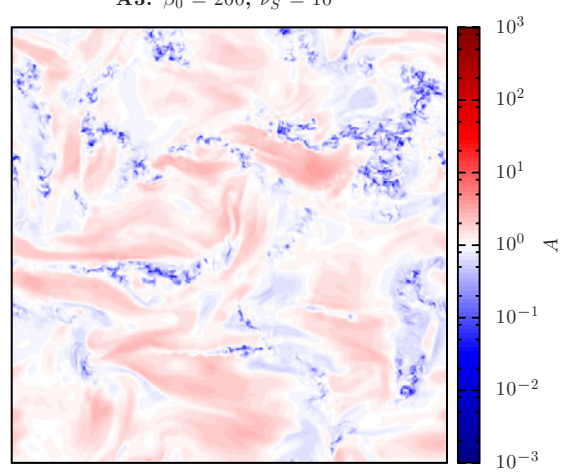

A1: $\beta_{0}=200, \nu_{S}=\infty$

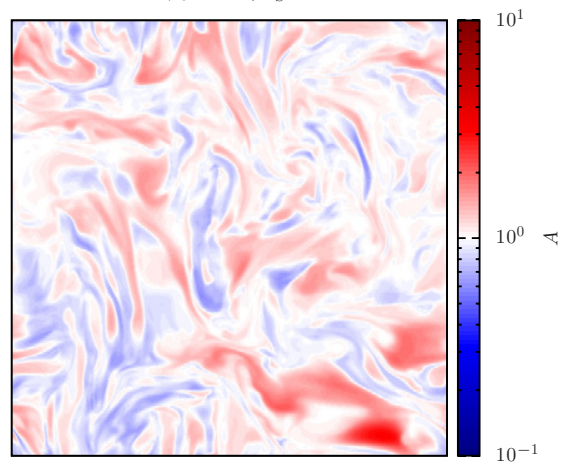

A4: $\beta_{0}=200, \nu_{S}=10^{2}$

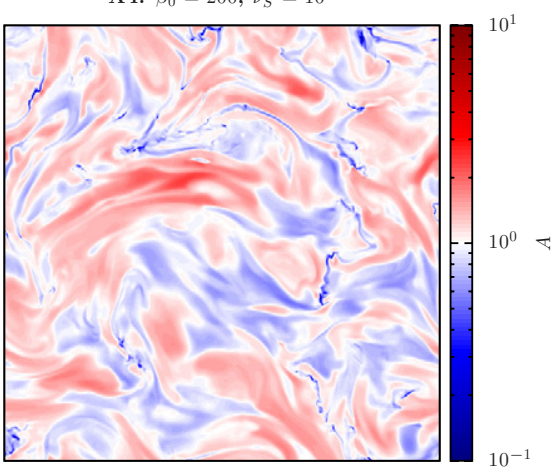

B1: $\beta_{0}=0.2, \nu_{S}=\infty$

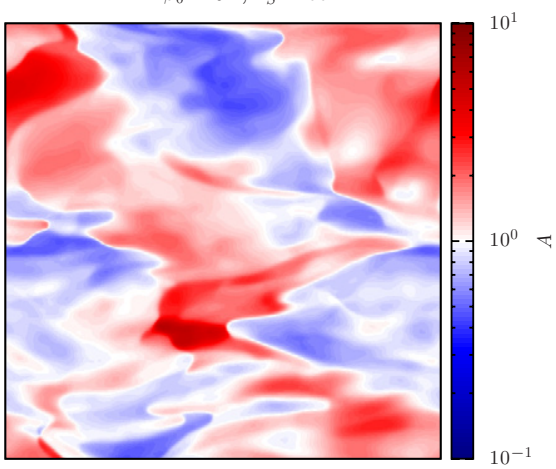

Figure 3. Maps of the anisotropy $A=p_{\perp} / p_{\|}$distribution at the central slice in the $X Y$ plane at the final time $t_{f}$ for a few models A and B of Table 1 .

(A color version of this figure is available in the online journal.)

(and also in local enhancements of density provided by parallel shocks). The correspondence of the low-intensity magnetic field with firehose unstable regions can be checked directly in model A 2 by comparing the maps of Figures 4 and 1 . The firehose unstable regions in models A2 and A3 in Figure 4 are small and fragmented, while in models A4 and A5 they are elongated (at lengths of the turbulent injection scale) and very thin (with thickness of the order of the dissipative scales) and are regions with magnetic field reversals and reconnection.

Also, from Figure 4 we see that most of the volume of models $\mathrm{A} 2$ and A3 is mirror unstable; for models A4 and A5, the mirror unstable regions are elongated but with much larger thickness than in the firehose unstable regions. We must remember that the criterion for the mirror unstable regions in Figure 4 is the kinetic one (Equation (9)) rather than the CGL-MHD criterion (Equation (8); see also Figure 2).

The spatial dimensions of the unstable regions in Figure 4 also reveal the maximum wavelength of the unstable modes that should develop inside the turbulent domain. In the models with finite anisotropy relaxation rate $v_{S}$, the larger the value of $v_{S}$, the smaller the wavelength of the unstable modes. For realistic values of $v_{S}$ of the order of $\gamma_{\max }$ (the maximum frequency of the instabilities), there would have only been unstable modes with wavelengths below the spatial dimensions we can resolve.

\subsection{Magnetic versus Thermal Stresses}

The gyrotropic tensor gives the gas a larger (smaller) strength to resist against bending or stretching of the field lines if $A>1$ $(A<1)$. This higher or smaller strength comes from the parallel anisotropic force

$$
f_{A}=\left(p_{\|}-p_{\perp}\right) \nabla_{\|} \ln B
$$

where $\nabla_{\|} \equiv(\mathbf{B} / B) \cdot \nabla$. The relative strength between this anisotropic force and the usual Lorentz curvature force can be estimated from $\alpha \equiv\left(p_{\|}-p_{\perp}\right) /\left(B^{2} / 4 \pi\right)$.

As a measure of the dynamical importance of the anisotropy, we calculated the average value of $|\alpha|$ for all the models of Table 1, and the values are listed in Tables 2, 3, and 4 for models $\mathrm{A}, \mathrm{B}$, and $\mathrm{C}$, respectively.

First, let us consider the models with initial moderate magnetic field $\left(\beta_{0}=200\right)$. For models A1, A6, A7, and A8 $\left(v_{S}=\infty\right)$, the anisotropic force is nondominant: $\langle|\alpha|\rangle \approx 0.4$. For model A2 $\left(v_{S}=0\right)$, on the other hand, the anisotropic force is dominant, with $\langle|\alpha|\rangle \approx 5$. For models A3, A4, and A5, with finite isotropization rate, the anisotropic force is comparable to the curvature force, being smaller for the higher isotropization rate: $\langle|\alpha|\rangle \approx 4$ for model A3 $\left(v_{S}=10^{1}\right)$ and $\langle|\alpha|\rangle \approx 1.5$ for model A5 $\left(v_{S}=10^{3}\right)$.

For the model with strong magnetic field $\left(\beta_{0}=0.2\right)$, model $\mathrm{B} 1$, the anisotropic force is negligible compared to the Lorentz curvature force: $\langle|\alpha|\rangle \approx 0.04$.

\subsection{PDF of Density}

Figure 5 shows the normalized histograms of $\log \rho$ for models A and B of Table 1 having different rates of anisotropy relaxation $v_{S}$. The upper panel shows models with initial moderate magnetic field intensity $\left(\beta_{0}=200\right)$, and the lower panel the model with initial strong magnetic field intensity $\left(\beta_{0}=0.2\right)$. The corresponding collisional MHD models are also shown for comparison.

Examining the high $\beta$ models in the top diagram, we note that all the models with anisotropy relaxation have similar distribution to the collisional model. Model A2, for which the anisotropy relaxation is null, has a much broader distribution, 

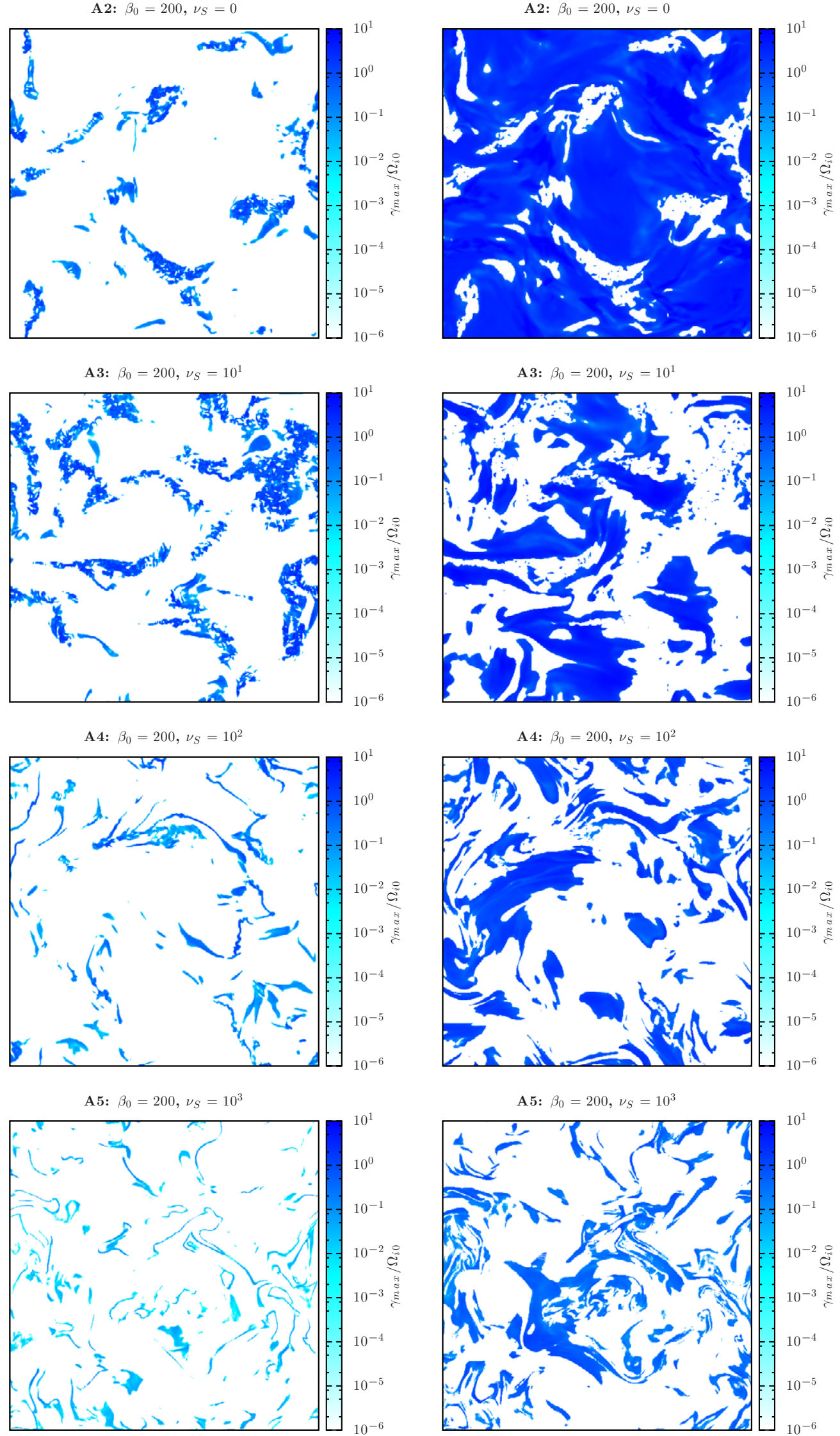

Figure 4. Central slice in the $X Y$ plane of the domain showing distributions of the maximum growth rate $\gamma_{\max }$ (normalized by the initial ion gyrofrequency $\Omega_{i 0}$ ) of the firehose (left column) and mirror (right column) instabilities for models A (with $\beta_{0}=200$ and different values of the anisotropy relaxation rate $v_{S}$ ). The expressions for the maximum growth rates are given by Equations (10), with a maximum value given by $\gamma_{\max } / \Omega_{i}=1$. Data are taken at the final time $t_{f}$ for each model, indicated in Table 1.

(A color version of this figure is available in the online journal.) 

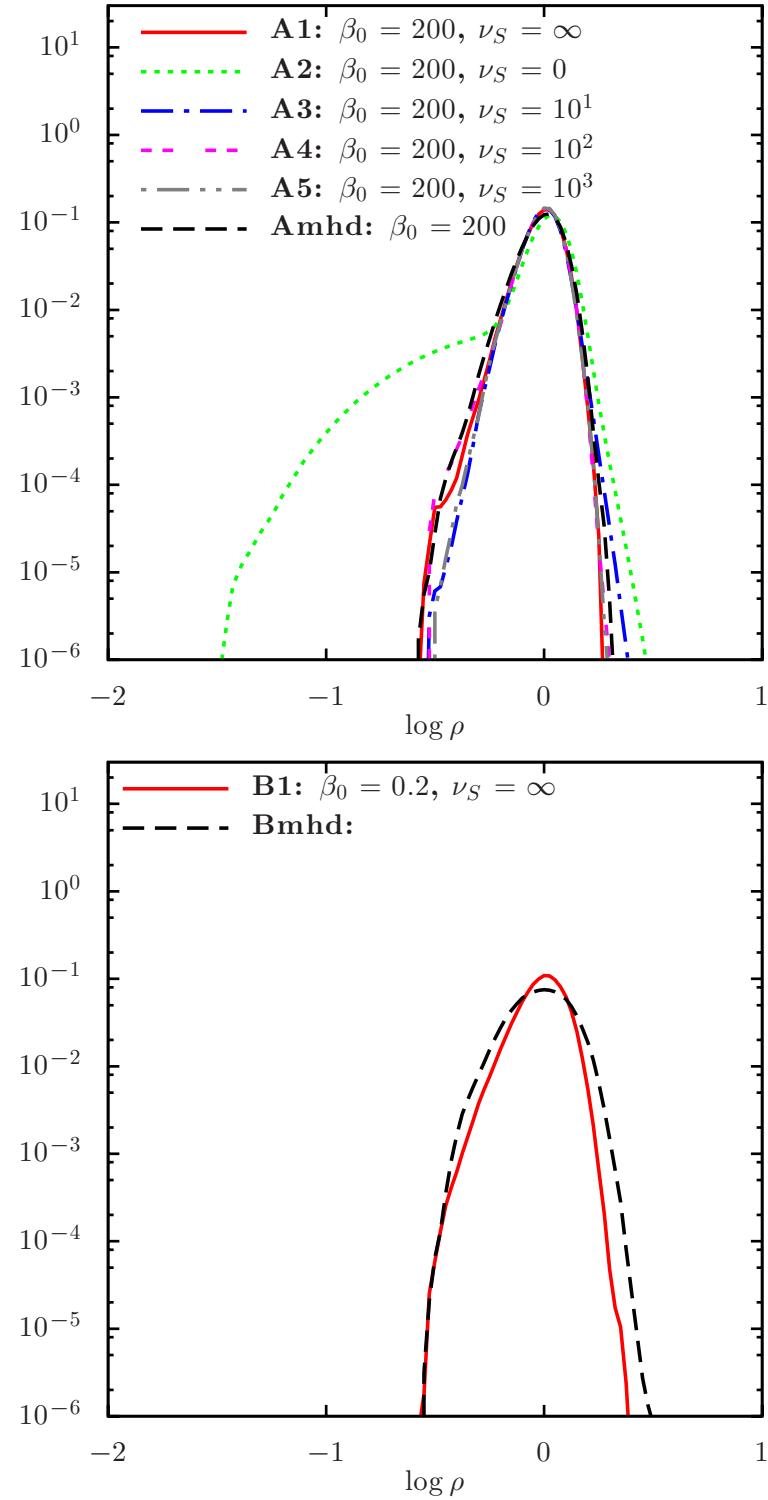

Figure 5. Normalized histogram of $\log \rho$. Top: models starting with $\beta_{0}=200$. Bottom: models starting with $\beta_{0}=0.2$. The histograms were calculated using one snapshot every $\Delta t=1$, from $t=2$ until the final time $t_{f}$ indicated in Table 1 .

(A color version of this figure is available in the online journal.)

especially in the low-density domain. This difference is due to the presence of strong mirror forces in the A2 model, which expels the gas to outside of high magnetic field intensity regions, causing the formation of low-density zones. Consistently, we can check this effect in the bi-histograms of density versus magnetic field intensity in Figure 10 of the Appendix for model A2 (the bi-histogram for model Amhd is also shown for comparison). The lowest density points are correlated with high-intensity magnetic fields for the model A2.

The bottom panel of Figure 5 indicates that the low- $\beta$, strong magnetic field model B1 has density distribution only slightly narrower than the collisional MHD model Bmhd, especially at the high-density region. The slight difference with respect to the collisional model is possibly due to: (1) the sound speed parallel to the field lines is higher in the collisionless models, $c_{\| s}=$ $\sqrt{3 p_{\|} / \rho}$ for the collisionless model, while for the collisional model $c_{s}=\sqrt{5 p / 3 \rho}$; and (2) in the direction perpendicular to the magnetic field, the fast modes have characteristic speeds higher in the collisionless model: $c_{f}=\sqrt{B^{2} / 4 \pi \rho+2 p_{\perp} / \rho}$, while for the MHD model $c_{f}=\sqrt{B^{2} / 4 \pi \rho+5 p / 3 \rho}$. These larger speeds in the anisotropic model imply a larger resistance to compression and therefore smaller density enhancements (at least for our transonic models).

\subsection{The Turbulence Power Spectra}

Power spectrum is an important characteristic of turbulence. For MHD turbulence substantial progress has been achieved recently as the Goldreich-Sridhar model has become acceptable. Recent numerical work has tried to resolve the controversies and confirmed the Kolmogorov - 5/3 spectrum of Alfvénic turbulence predicted in the model (e.g., Beresnyak \& Lazarian 2009, 2010; Beresnyak 2011, 2012b). This spectrum corresponds to the Alfvénic mode of the compressible MHD turbulence (Cho \& Lazarian 2002, 2003; Kowal \& Lazarian 2010; Beresnyak \& Lazarian 2013).

Our goal here is to determine the power spectrum of the turbulence in collisionless plasma in the presence of the feedback of plasma instabilities on scattering.

Figure 6 compares, for different models of Table 1, the power spectra of the velocity (top row), magnetic field (middle row), and density (bottom row). The models starting with moderate magnetic field and $\beta_{0}=200$ (A1, A2, A3, A4, A5, Amhd), for which the turbulence is super-Alfvénic, are in the left column, and the models starting with strong magnetic field and $\beta_{0}=0.2$ (B1, Bmhd), for which the turbulence is sub-Alfvénic, are in the right column. Each power spectrum is multiplied by the factor $k^{5 / 3}$

The velocity power spectrum $P_{u}(k)$ for the super-Alfvénic high- $\beta$ collisional model Amhd (in the left top panel of Figure 6) is consistent with the Kolmogorov slope approximately in the interval $4<k<20$ and decays quickly for $k>30$. The power spectra of the collisionless models A1, A3, A4, and A5 are similar, but show slightly less power in the interval $4<k<30$. In fact, in Table 2 in the Appendix, we find that the average values of $u^{2}$ for these models are smaller than the model Amhd. Models A3 and A4 evidence more power at the smallest scales, already at the dissipation range. This is due to the acceleration of gas produced by the firehose instability (see Figure 2). Model A2 ( $\left.v_{S}=0\right)$ has a flatter velocity power spectrum than the collisional MHD model Amhd and much more power at the smallest scales. This excess of power comes from the firehose and mirror instabilities and is consistent with the trend reported in the previous sections and also in Kowal et al. (2011b).

The sub-Alfénic velocity power spectrum $P_{u}(k)$ of the collisional MHD model Bmhd (top right panel in Figure 6) has a narrower interval of wavenumbers consistent with the Kolmogorov slope. The power spectra $P_{u}(k)$ of the collisionless model B1 are almost identical, which is in agreement with the small dynamical importance of the anisotropy forces compared to the magnetic forces (see Section 4.2).

The power spectrum related to the compressible component of the velocity field $P_{C}(k)$ is shown in Figure 11 in the Appendix, where it is divided at each wavenumber by the total power of the velocity field. For the high- $\beta$ models, the ratio $P_{C}(k) / P_{u}(k)$ for the collisionless models is similar to that of the collisional MHD model Amhd for almost every wavenumber $k$ and is $\approx 0.15$. For the low- $\beta$ model, however, the collisionless model has a ratio $P_{C}(k) / P_{u}(k)$ slightly higher than that of the collisional MHD model Bmhd for wavenumbers above $k \approx 10$. The fractional 

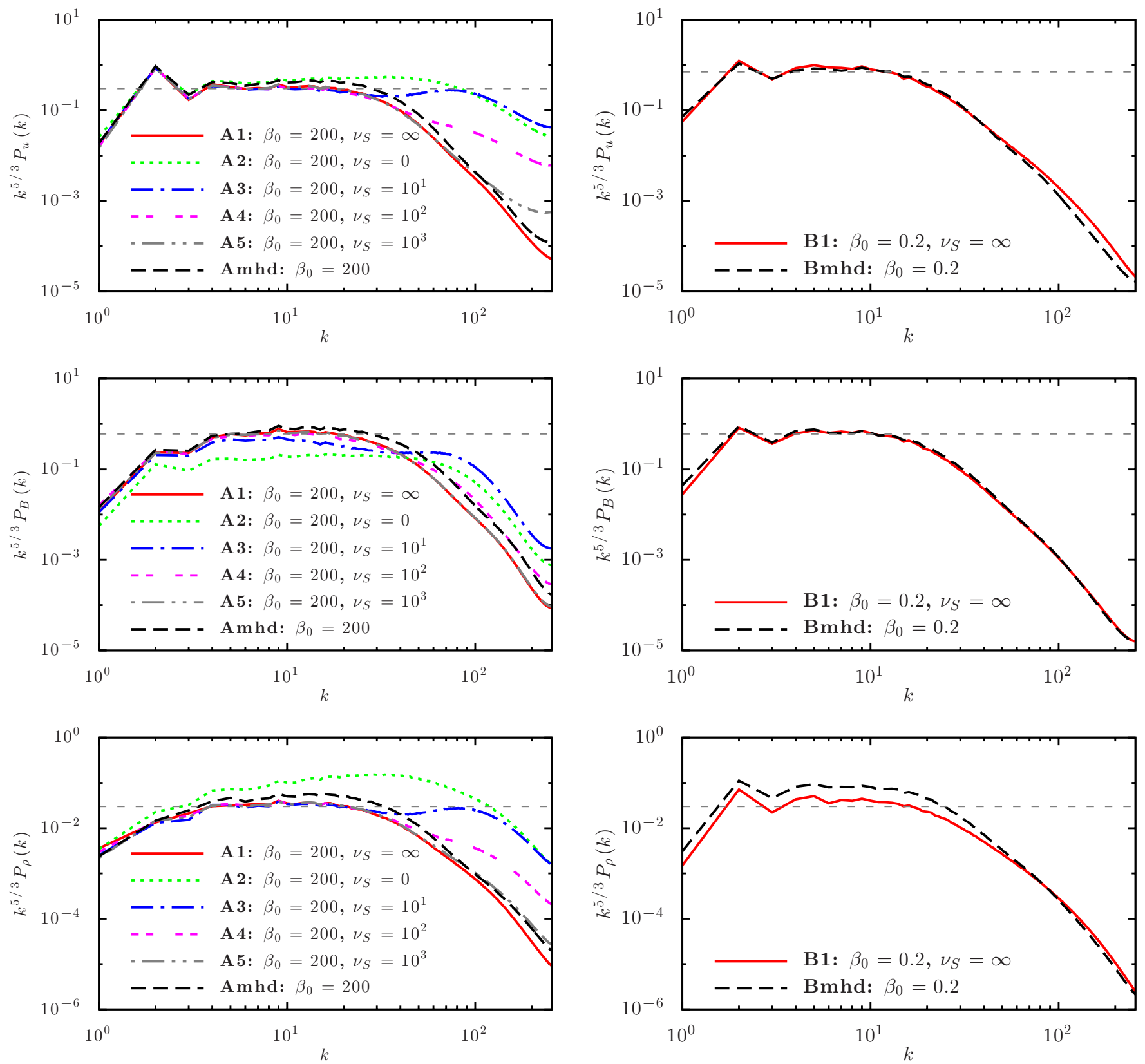

Figure 6. Power spectra of the velocity $P_{u}(k)$ (top row), magnetic field $P_{B}(k)$ (middle row), and density $P_{\rho}(k)$ (bottom row), multiplied by $k^{5 / 3}$. Left column: models $\mathrm{A}$, with initial $\beta_{0}=200$. Right column: models $\mathrm{B}$, with $\beta_{0}=0.2$. Each power spectrum was averaged in time considering snapshots every $\Delta t=1$, from $t=2$ to the final time step $t_{f}$ indicated in Table 1.

(A color version of this figure is available in the online journal.)

power in the compressible modes in the interval $2<k<20$ is smaller compared to the super-Alfvénic (high- $\beta$ ) models, but at larger wavenumbers it becomes higher.

The anisotropy in the structure function of the velocity is shown in Figure 7. The structure function of the velocity $S_{2}^{u}$ is defined by

$$
S_{2}^{u}\left(l_{\|}, l_{\perp}\right) \equiv\left\langle|\mathbf{u}(\mathbf{r}+\mathbf{l})-\mathbf{u}(\mathbf{r})|^{2}\right\rangle,
$$

where the displacement vector $\mathbf{I}$ has the parallel and perpendicular components (relative to the local mean magnetic field) $l_{\|}$and $l_{\perp}$, respectively. The local mean magnetic field is defined by $(\mathbf{B}(\mathbf{r}+\mathbf{l})+\mathbf{B}(\mathbf{r})) / 2$ (as in Cho et al. 2002 and Zrake \& MacFadyen 2012). The GS95 theory predicts an anisotropy scale dependence of the velocity structures (eddies) of the form $l_{\|} \propto l_{\perp}^{2 / 3}$. The axis in Figure 7 is in cell units. The collisional MHD model Amhd is consistent with the GS95 scaling for the interval $10 \Delta<l_{\perp}<40 \Delta$, where $\Delta$ is one cell unit in the computational grid. For the sub-Alfvénic model Bmhd, however, this scaling is less clear, although the anisotropy is clearly seen.

The collisionless models A1, A4, and A5 in Figure 7 evidence anisotropy in the velocity structures, which is identical to that of the collisional MHD model Amhd. Models A2 and A3, on the other hand, have more isotropized structures at small values of $l$. This effect is due to the action of the instabilities and is also observed in the high- $\beta$ models in Kowal et al. (2011b) for both the firehose and mirror instability regimes.

Using the two-point statistics of the structure function, we can also estimate the velocity power spectrum in the directions 

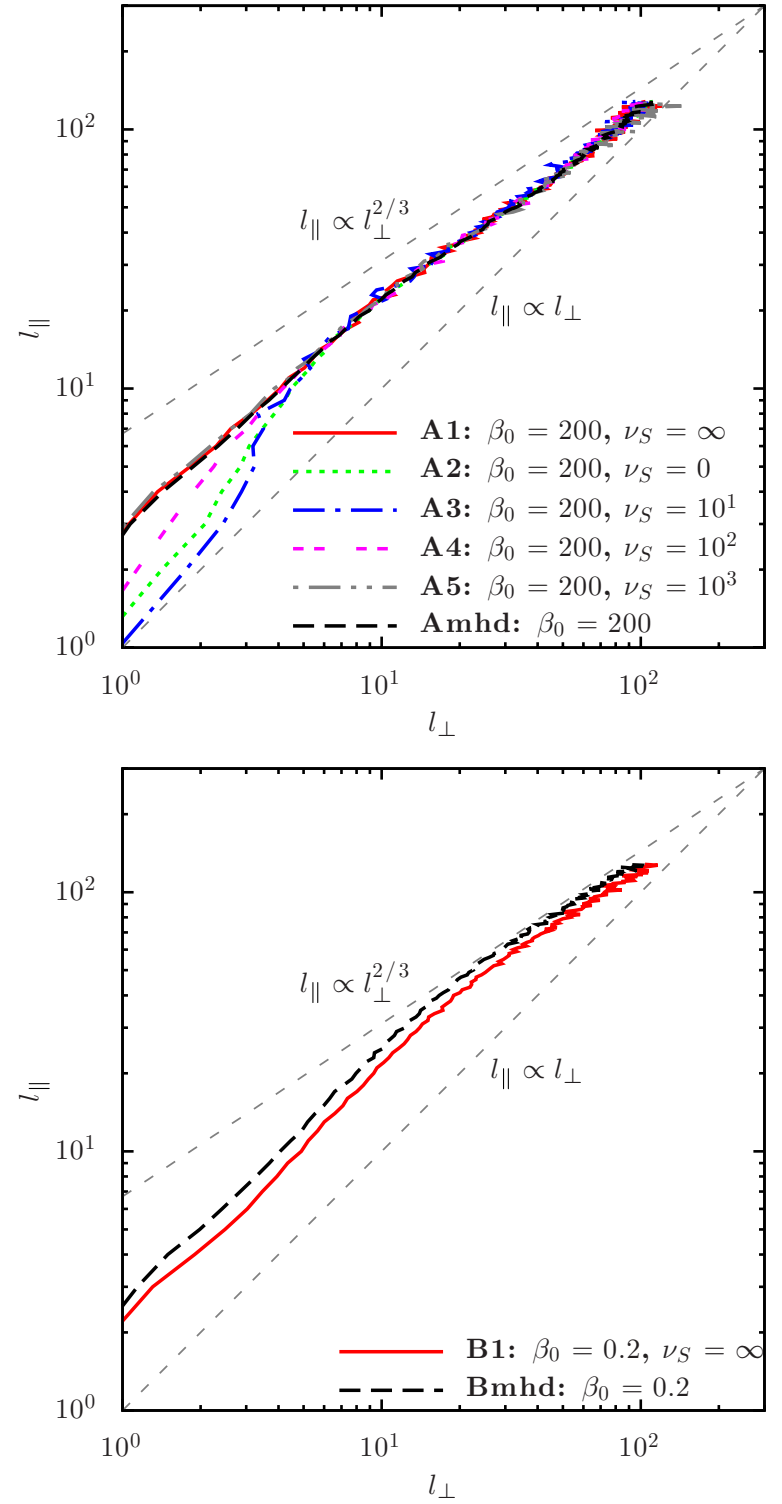

Figure 7. $l_{\perp}$ vs. $l_{\|}$obtained from the structure function of the velocity field (Equation (17)). The axes are scaled in cell units.

(A color version of this figure is available in the online journal.)

parallel or perpendicular to the local mean magnetic field (see Cho et al. 2002 for a detailed discussion on this subject). The scaling laws $S_{2}^{u}\left(l_{\|}, 0\right) \propto l_{\|}^{\zeta}$ and $S_{2}^{u}\left(0, l_{\perp}\right) \propto l_{\perp}^{\xi}$ are related to the one-dimensional power spectra in the directions parallel and perpendicular to the local mean magnetic field: $E\left(k_{\|}\right) \propto k_{\|}^{-(1+\zeta)}$ and $E\left(k_{\perp}\right) \propto k_{\perp}^{-(1+\xi)}$, respectively. For the range of scales corresponding to the wavenumbers $4<k<20$, we fitted $\zeta$ and $\xi$ for the different models. The results are shown in Table 5 (we should observe that this fitting is sensitive to the range of scales chosen). For the model Amhd, our results are close to the expectations of the GS95 theory: $E\left(k_{\perp}\right) \propto k_{\perp}^{-5 / 3}$ and $E\left(k_{\|}\right) \propto k_{\|}^{-2}$ (also in agreement with the values obtained by Cho et al. 2002). Also, at least for the velocity field, the powerlaw indices of the collisionless models A1-A5 in Table 5 are similar to model Amhd, except for model A2, for which the values of $\zeta$ and $\xi$ result in flatter power-law spectra.

The magnetic field power spectra $P_{B}(k)$ of the collisional MHD models Amhd and Bmhd (middle row of Figure 6) show a power law consistent with the Kolmogorov slope at the same intervals of the velocity power spectra. As in the velocity power spectrum, in the high- $\beta$, super-Alfvénic cases, the collisionless models A1, A3, A4, and A5 have similar $P_{B}(k)$ to the collisional model Amhd (although with slightly less power). Model A2 has a $P_{B}(k)$ much flatter than that of Amhd and has less power (by a factor of two) at the inertial range interval. In the smallest scales $(k>50)$, however, its power is above that of the Amhd model (this is also observed for model A3). As in the velocity power spectrum, these small-scale structures are due to the instabilities that are present in this model.

For the sub-Alfvénic, low- $\beta$ models (B), the magnetic field power spectrum $P_{B}(k)$ of the collisionless model is again similar to the collisional MHD model Bmhd.

Figure 12 of the Appendix compares $P_{B}(k)$ and $P_{u}(k)$ for our models. For the super-Alfvénic, high- $\beta$ models (A) that are in steady state, the magnetic field power spectrum is in super equipartition with the velocity power spectrum for $k>3$ for all models, except the A2 model, which has $P_{B}(k)<P_{u}(k)$ for all wavenumbers. Models A3, A4, and A5 show $P_{B}(k) / P_{u}(k)$ decreasing values for larger wavenumbers, this effect being more pronounced in model A3, which has a smaller anisotropy relaxation rate. The sub-Alfvénic, low- $\beta$ collisionless model B1 has the ratio $P_{B}(k) / P_{u}(k)$ slightly smaller than unity for all wavenumbers and slightly smaller than the collisional Bmhd model at large $k$ values.

The anisotropy in the structure function for the magnetic field shows a similar trend to the velocity field in all models and is not presented here. Likewise, the density power spectra $P_{\rho}(k)$ for the super-Alfvénic, high- $\beta$ models (bottom row in Figure 6) reveal the same trend of the velocity power spectra. For the sub-Alfvénic model, however, the smaller power in the larger scales compared to the collisional MHD model Bmhd is clearly evident, specially in the inertial range. This is consistent with the discussion following the presentation of the density distribution (Section 4.3), which evidenced that the collisionless models resist more to compression than the collisional model (see also Figure 11).

\subsection{Turbulent Amplification of Seed Magnetic Fields}

Figure 8 shows the magnetic energy evolution of the models having initially very weak magnetic (seed) field, models C1, C2, C3, C4, and Cmhd of Table 1. The kinetic energy of the models is not shown, but their values are approximately constant in time (after $t \approx 1$ ), and their average values (taken during the last $\Delta t=10$ for each model) $\left\langle E_{K}\right\rangle$ are shown in Table 4. For each of these models, Figure 9 shows the power spectrum of the magnetic field, from $t=2$ until the final time, for every $\Delta t=2$ (dashed lines). The final magnetic field power spectrum is the continuous line. Also for comparison, the final velocity power spectrum is plotted (dash-dotted line).

The collisional MHD model Cmhd shows an initial exponential growth of the magnetic energy until $\mathbf{t} \approx 5$. In this interval, the average magnetic energy grows from $E_{M}=5 \times 10^{-7}$ to $E_{M} \sim 10^{-2}$. After this, a slower (linear) growth rate of the magnetic energy takes place until $t \approx 10$, as can be seen in the bottom panel of Figure 8. This is consistent with studies of turbulent dynamo amplification of magnetic fields in collisional plasmas (see, e.g., Cho et al. 2009). At the final times, the magnetic energy achieves the value $E_{M} \approx 9.0 \times 10^{-2}$ which is approximately four times smaller than the average kinetic energy $E_{K} \approx 0.38$ (see Table 4). The bottom panel of Figure 9 shows that the final magnetic field power spectrum is peaked 

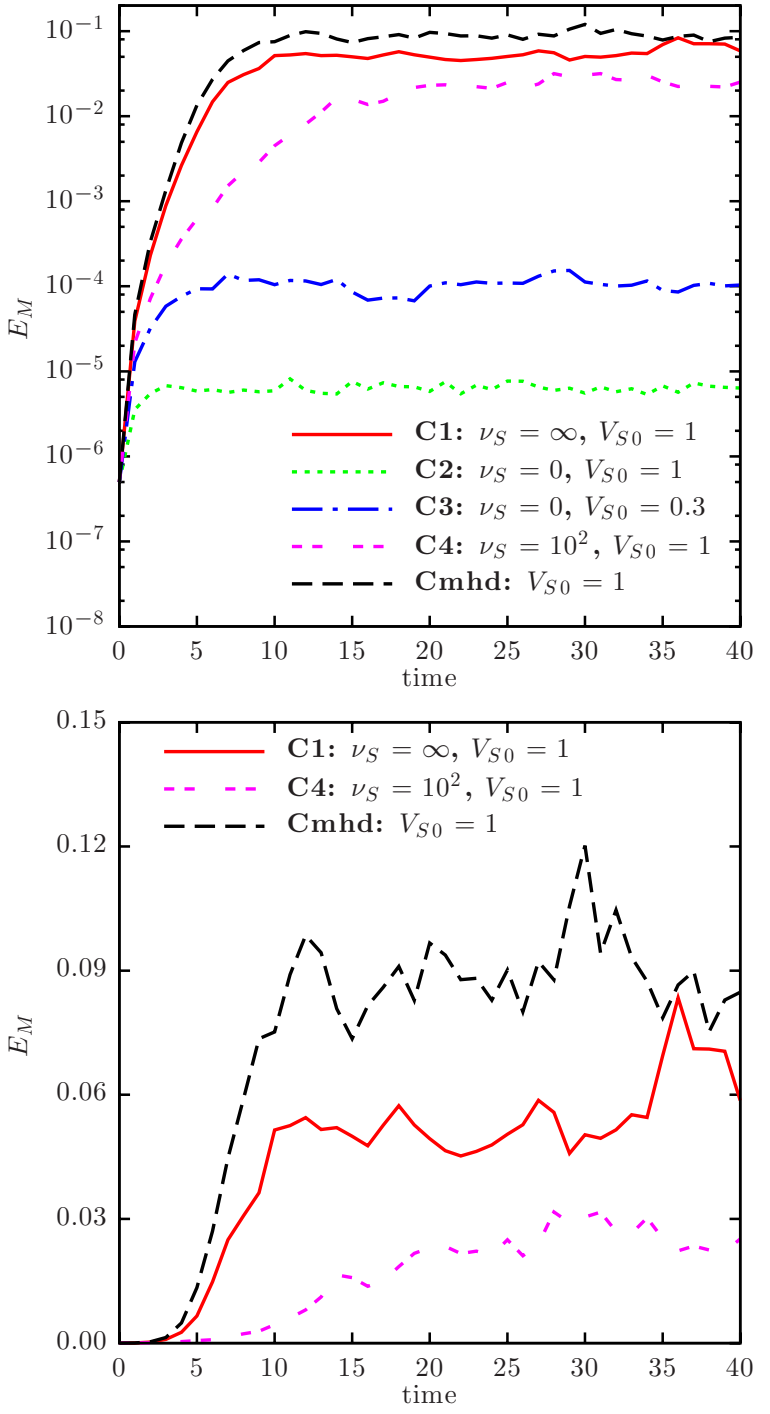

Figure 8. Time evolution of the magnetic energy $E_{M}=B^{2} / 2$ for the models starting with a weak (seed) magnetic field, models C1, C2, C3, C4, and Cmhd, from Table 1. The bottom and top panels differ only in the scale of $E_{M}$. This is shown in a log scale in the top panel and in a linear scale in the bottom panel. The curves corresponding to models $\mathrm{C} 2$ and $\mathrm{C} 3$ are not visible in the bottom panel.

(A color version of this figure is available in the online journal.)

at $k \approx 20$ above, which it is in super-equipartition with the velocity power spectrum.

The collisionless model $\mathrm{C} 1$ with instantaneous relaxation of the pressure anisotropy $\left(v_{S}=\infty\right)$ has a turbulent amplification of the magnetic energy very similar to that of the collisional MHD model Cmhd (Figure 8). The initial exponential growth rates are nearly indistinguishable between the two models, but in the linear stage the growth rate is slightly smaller in model C1 (see bottom panel of Figure 8 ) and also the final value of saturation of the magnetic energy: $E_{M} \approx 6.2 \times 10^{-2}$ (see Table 4). During the initial exponential growth of the magnetic energy, when the plasma still has high values of $\beta$, the pressure anisotropy relaxation due to the kinetic instabilities keeps the plasma mostly isotropic, explaining the similar behavior to the collisional MHD model. When $\beta$ starts to decrease, the anisotropy $A$ can increase (or decrease), spanning a range of $A$ values in the stable zone (as in Figure 2 for model A1). Then, the anisotropic forces can start to have dynamical importance. At the final times, the value of $\left\langle\left|p_{\|}-p_{\perp}\right| /\left(B^{2} / 4 \pi\right)\right\rangle$, which measures the dynamical importance of the anisotropic forces compared to the Lorentz curvature force (see Section 4.2), is $\approx 0.5$ (Table 4). The magnetic field power spectrum has an identical shape to the model Cmhd, specially in the final time step.

The turbulent dynamo is also tested for a model with a finite anisotropy relaxation rate, model $\mathrm{C} 4$, which has $v_{S}=10^{2}$. The growth rate of the magnetic energy (in the exponential and linear phases) is smaller compared to models Cmhd and C1 (top and bottom panels in Figure 8). In this case, the anisotropy $A>1$ develops moderately during the magnetic energy amplification and gives the mirror forces some dynamical importance to change the usual collisional MHD dynamics. The value of the magnetic energy at the final time of the simulation is approximately one-third of the value for the collisional MHD model. The final magnetic power spectrum has a shape similar to the collisional MHD model Cmhd, but below the equipartition with the velocity field power spectrum, which has more power at the smallest scales due to the presence of the instabilities (Figure 9).

Model C2, a standard CGL model with no constraints on the growth of pressure anisotropy $\left(v_{S}=0\right)$, shows no evidence of a turbulent dynamo amplification of its magnetic energy that saturates at very low values already at $t \approx 5$ (Figure 8 ), when $E_{M} \approx 6.2 \times 10^{-6}$, while the kinetic energy is $E_{K} \approx 0.32$ (see Table 4). The reason is that the anisotropy $A$ increases at the same time that the magnetic field is increased $\left(A \propto B^{3} / \rho^{2}\right.$ in the CGL closure), giving rise to strong mirror forces along the field lines, which increase their resistance against bending or stretching. For this model, $\left\langle\left|p_{\|}-p_{\perp}\right| /\left(B^{2} / 4 \pi\right)\right\rangle \sim 10^{5}$, that is, the anisotropic forces dominate over the Lorentz force. The magnetic field power spectrum (top right panel in Figure 9) is similar in shape (but not in intensity) to the Cmhd model, being peaked at $k \approx 40$.

The saturated value of the magnetic energy for models without anisotropy relaxation is, nevertheless, sensitive to the initial plasma $\beta$. Model $\mathrm{C} 3$ is similar to model $\mathrm{C} 2$, but starts with a lower sound speed $\left(V_{S 0}=0.3\right)$, which makes $\beta 10$ times smaller (see Table 1). Turbulence is supersonic in this case, rather than transonic. The magnetic energy evolution is similar to that of model $\mathrm{C} 2$, but the magnetic energy saturates with a value about two orders of magnitude larger, although the anisotropic forces are still dominant, with $\left\langle\left|p_{\|}-p_{\perp}\right| /\left(B^{2} / 4 \pi\right)\right\rangle \sim 10^{4}$ (see Table 4).

\section{DISCUSSION}

The anisotropy in pressure created by the turbulent motions gives rise to new forces in the collisionless MHD description (see the momentum conservation equation in Equation (1)). These new forces gain dynamical importance when the anisotropy $A=p_{\perp} / p_{\|}$deviates significantly from unity (depending on $\beta$ ) and give rise to instabilities. The standard CGL-MHD model is able of capturing the correct linear behavior of the long-wavelength limit of the firehose instability (which has scales much larger than the proton Larmor radius $l_{c p}$ ), but not of the mirror instability, which is overstable (see the kinetic and CGL-MHD instability limits in the $A-\beta_{\|}$plane in Figure 2). The correct linear threshold of the mirror instability can be obtained from higher order fluid models that evolve heat conduction (e.g., Snyder et al. 1997; Ramos 2003; Kuznetsov $\&$ Dzhalilov 2010) and results in substantial difference with regard to the CGL-MHD criterion (see the kinetic and CGL-MHD instability limits in the $A-\beta_{\|}$plane in Figure 2). 

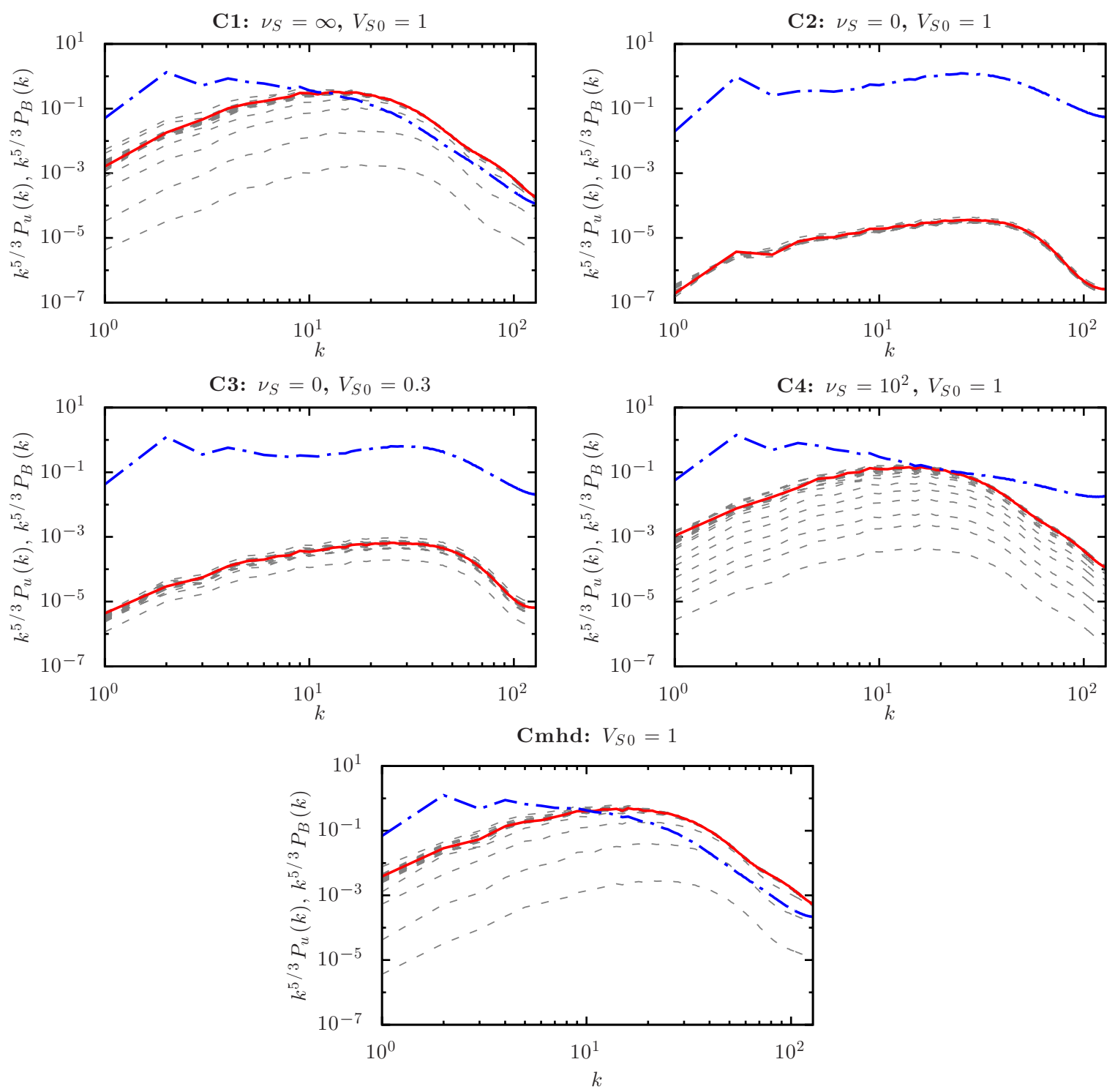

Figure 9. Magnetic field power spectrum multiplied by $k^{5 / 3}$ for the same models presented in Figure 8 , from $t=2$ at every $\Delta t=2$ (dashed lines) until the final time indicated in Table 1 (solid lines). The velocity field power spectrum multiplied by $k^{5 / 3}$ at the final time is also depicted for comparison (dash-dotted line).

(A color version of this figure is available in the online journal.)

These same (mirror and firehose) instabilities are known to constrain the (proton) pressure anisotropy growth to values close to the instability thresholds, via wave-particle interactions that obviously are not captured by any fluid model. Other kinetic instabilities driven by pressure/temperature anisotropy are also known to relax the anisotropy, such as the cyclotron instability (for protons) and whistler anisotropy instability (for electrons; see Gary 1993). Based on this phenomenology, we here imposed source terms on the standard CGL-MHD equations that relaxed the pressure anisotropy $A$ to the marginally stable value (conserving the internal energy) at a rate $v_{S}$, whenever $A$ evolved to a value inside the unstable kinetic mirror or firehose zones.

As remarked before, there are several studies about the rate at which instabilities driven by pressure anisotropy relax the anisotropy itself. Using 2D particle simulations, Gary et al. (2000) studied the anisotropy relaxation rate for protons subject to cyclotron instability and found rates that are related to the growth rate of the fastest unstable mode $\sim 10^{-3}-10^{-1} \Omega_{p}$ (where $\Omega_{p}$ is the proton gyrofrequency). Nishimura et al. (2002), also employing 2D particle simulations, found an analogous result for electrons subject to the whistler anisotropy instability with an anisotropy relaxation rate of a few percent of the electron gyrofrequency. In both studies, part of the free energy of the instabilities is converted to magnetic energy. Recently, Yoon \& Seough (2012) and Seough \& Yoon (2012) studied the saturation of specific modes of the mirror and firehose instabilities via quasi-linear calculations, using the Vlasov-Maxwell dispersion relation. They also found that the temperature anisotropy relaxes to the marginal state after a few hundreds of the proton Larmor period and there is accumulation of magnetic fluctuations at the proton Larmor radius scales.

However, exactly what kinetic instabilities saturate the pressure anisotropy or the detailed processes involved are not fully understood yet, and one cannot be sure to what extent the rates inferred in the studies above or those employed in the present analysis are applicable to the ICM plasma, especially with driven turbulence. In other words, the rate $v_{S}$ is subject to uncertainties, and further forthcoming study involving PIC simulations will be 
performed in order to investigate this issue in depth. In particular, in a very recent study about accretion disks, Riquelme et al. (2012) performed direct 2D PIC shearing box simulations and found that for low- $\beta$ values $(\beta<0.3)$, the pressure anisotropy is constrained by the ion-cyclotron instability threshold, while for high- $\beta$ values the mirror instability threshold constrains the anisotropy, which is compatible with the present study. However, they have also found that in the low- $\beta$ regime, initially the anisotropy can reach maximum values above the threshold due to the mirror instability. Nevertheless, they have attributed this behavior to the initial cyclotron frequency adopted for the particles, which was small compared to the orbital frequency in order to save computation time.

We must add yet that here we have taken into account the isotropization feedback due to the firehose and mirror instabilities only, neglecting, for instance, the ion-cyclotron instability because this is more likely to be important in low$\beta_{\|}$regimes, which is not the case for ICM plasmas. We have considered that the anisotropy relaxation to the marginally stable value occurs at the rate of the fastest mode of the triggered instability (Equations (10)), which is of the order of the proton gyrofrequency. As discussed above, for the typical parameters of the ICM, these relaxation times correspond to timescales that are extremely short compared to the shortest dynamical times that one can solve. This means that the plasma at least at the macroscopic scales is essentially always inside the stable region. This justifies why we adopted the simple approach of constraining the anisotropy by the marginal values of the instabilities, similarly to the hardwall constraints employed by Sharma et al. (2006). However, if one could resolve all the scales and frequencies of the system, one would probably detect some fraction of the plasma at the small scales lying in the unstable region. For the ICM, the scale of the fastest-growing mode is $\sim 10^{10} \mathrm{~cm}$, i.e., the proton Larmor radius.

\subsection{Consequences of Assuming One-temperature Approximation for All Species}

Although the electrons have a larger collisional rate than the protons in the ICM $\left(\sim \sqrt{m_{p} / m_{e}}\right)$, we have assumed in this work, for simplicity, that both species have the same anisotropy in pressure. Also, we assumed them to be in "thermal equilibrium." A more precise approximation would be to consider the electrons only with an isotropic pressure. This would require another equation to evolve the electronic pressure and additional physical ingredients in our model, such as a prescription on how to share the turbulent energy converted into heat at the end of the turbulent cascade or how to quantify the thermalization of the free-energy released by the kinetic instabilities, as well as a description of the cooling for each of the species. The assumption of same temperature and pressure anisotropy for both species has resulted in a force on the collisionless plasma due to the latter that is maximized. Nevertheless, since our results have shown that the dynamics of the turbulence when considering the relaxation of the anisotropy due to the instabilities feedback is similar to that of collisional MHD, we can conclude that if we had considered the electronic pressure to be already isotropic, then this similarity would be even greater.

Another relevant aspect that should be considered in future work regards the fact that the electron thermal speed achieves relativistic values for temperatures $\sim 10 \mathrm{keV}$, which are typical in the ICM. Thus, a more consistent calculation would require a relativistic treatment (see, for example, Hazeltine \& Mahajan 2002).

\subsection{Limitations of the Thermal Relaxation Model}

Our model considers a thermal relaxation (Equation (13)), which ensures that the average temperature of the domain is maintained nearly constant, despite the continuous dissipation of turbulent power. This simplification allowed us to avoid a detailed description of the radiative cooling and its influence on the temperature anisotropy, even though the rate $v_{\text {th }}=5$ employed in most of our simulations is low enough to not perturb significantly the dispersion relation arising from the CGL-MHD equations. Timescales $\delta t \simeq v_{\text {th }}^{-1}=0.2$ are much larger than the typical time step of our simulations $\left(\sim 10^{-5}\right)$. This means that the maximum characteristic speeds calculated via relations (5) and (6) were more than appropriate for the calculation of the fluxes in our numerical scheme (see Section 3.1).

In order to evaluate the effects of the rate of the thermal relaxation on the turbulence statistics, we also performed numerical simulations of three models with different rates $v_{\text {th }}$ (namely, models A6, A7, and A8 of Table 1). Model A6 has no thermal relaxation $\left(v_{\text {th }}=0\right)$, while model A7 has a slower rate than model $\mathrm{A} 1, \nu_{\text {th }}=0.5$, and both $\mathrm{A} 6$ and $\mathrm{A} 7$ systems undergo a continuous increase of the temperature as time evolves, which increases $\beta$ and reduces the sonic Mach number of the turbulence. Model A8, on the other hand, has a faster rate $v_{\text {th }}=50$ and quickly converges to the isothermal limit. Despite different averages and standard deviations in their internal energy, models A6, A7, and A8 presented overall behavior similar to model A1 (see Table 2).

We have also tested models without anisotropy relaxation (not shown here) that employed the CGL-MHD equations of state for calculating the pressure components parallel and perpendicular to the magnetic field (Equations (4) accompanied by homogeneous initial conditions, rather than evolving the last two equations in Equation (1) for the anisotropy $A$ and the internal energy, respectively). Although there are some intrinsic differences due to larger local values of the sound speeds, the overall behavior of these models was qualitatively similar to the models with $v_{S}=0$ presented here.

In spite of the results above, a more accurate treatment of the energy evolution will be desirable in future work. For instance, as discussed earlier, the lack of a proper treatment for the heat conduction makes the linear behavior of the mirror instability in a fluid description different from the kinetic theory, leading to an overstability of the system. A higher order fluid model reproducing the kinetic linear behavior of the mirror instability (see Equation (9)) would enhance the effects of this instability in the models with finite $v_{S}$, probably producing more smallscale fluctuations compared to the present results (see Figure 4). The effects of the mirror instability on the turbulence statistics have been extensively discussed in Kowal et al. (2011b; see also next section), where a double-isothermal closure was used. This closure is able to reproduce the threshold of the mirror instability given by kinetic derivation.

\subsection{Comparison with Previous Studies}

Kowal et al. (2011b) studied the statistics of the turbulence in collisionless MHD flows assuming fixed parallel and perpendicular temperatures in the so-called double-isothermal approximation, but without taking into account the effects of anisotropy saturation due to the instability feedback. They explored different regimes of turbulence (considering different combinations of sonic and Alfvénic Mach numbers) and initially different (firehose or mirror) unstable regimes. They analyzed the power 
spectra of the density and velocity, and also the anisotropy of the structure function of these quantities, and found that superAlfvénic, supersonic turbulence in these double-isothermal collisionless models does not evidence significant differences compared to the collisional-MHD counterpart.

In the case of subsonic models, they have also detected an increase in the density and velocity power spectra at the smallest scales due to the growth of the instabilities at these scales, when compared to the collisional-MHD counterparts. They found elongation of the density and velocity structures along the magnetic field in mirror unstable simulations and isotropization of these structures in the firehose unstable models. In the present study, the closest to their models is the high $\beta$, super-Alfvénic model A2, which is without anisotropy relaxation. As in their subsonic sub-Alfvénic mirror unstable case, the instabilities accumulate power in the smallest scales of the density and velocity spectra. However, we should note that the density and velocity structures in our Model A2 become more isotropic at these scales probably because it is in a super-Alfvénic regime.

Our simulations starting with initial seed magnetic field have revealed the crucial role of the pressure anisotropy saturation (due to the mirror instability) for the dynamo turbulent amplification of the magnetic field, which in turn increases the anisotropy $A$. In our seed field simulations without anisotropy constraints (models C2 and C3), where the mirror forces dominate the dynamics, the turbulent flow is not able to stretch the field lines; therefore, there is no magnetic field amplification. This is consistent with earlier results presented in Santos-Lima et al. (2011) and de Gouveia Dal Pino et al. (2013), and also with the findings of de Lima et al. (2009), where the failure of the turbulent dynamo using a double-isothermal closure for $p_{\perp}>p_{\|}$was reported. On the other hand, in model C1 where the pressure anisotropy growth is constrained by the instabilities, there is a dynamo amplification of the magnetic energy until nearly equipartition with the kinetic energy. This result is in agreement with 3D numerical simulations of MRI turbulence performed by Sharma et al. (2006), where a collisionless fluid model taking into account the effects of heat conduction was employed in a shearing box. They have found that the anisotropic stress stabilizes the MRI when no bounds on the anisotropy are considered, making the magnetic lines stiff and avoiding its amplification. When using bounds on the anisotropy, however, they found that the generated MRI is similar (but with corrections of order of unity) to the collisional-MHD case. Sharma et al. (2006), however, did not consider any cooling mechanism, so that the temperature increased continuously in their simulations. Besides, the simulations presented here have substantially larger resolution. Further, they have found that the system overall evolution is nearly insensitive to the adopted threshold values for the anisotropy. We have also found little difference in the turbulence statistics between models with different non-null values of the anisotropy relaxation rate.

Meng et al. (2012a) also employed a collisionless MHD model to investigate the Earth's magnetosphere by means of 3D global simulations. They employed the CGL closure, adding terms to constrain the anisotropy in the ion pressure only (the electronic pressure considered isotropic was neglected in their study). Using real data from the solar wind at the inflow boundary, they compared the outcome of the model in trajectories where data from spacecraft (correlated to the inflow data) were available. Then, they repeated the same calculation, but employing a collisional MHD model. They found better agreement with the collisionless MHD model in the trajectory passing by the bowshock region, where gas is compressed in the direction parallel to the radial magnetic field lines, producing a firehose $(A<1)$ unstable zone. However, in the trajectory passing by the magnetotail, the simulated data in the collisionless model were not found to be more precise than in the collisional case. In summary, the collisionless MHD description of the magnetosphere seems to differ little from the standard MHD model when the anisotropy is constrained. Even though, they have found quantitative differences in, for example, the thickness of the magnetosheath, which is augmented in the collisionless case, in better agreement with the observations. In the more homogeneous problem discussed here, in a domain with periodic boundaries and isotropic turbulence driving, we have found that both the evolution of the turbulence and the turbulent dynamo growth in the ICM under a collisionless-MHD description accounting for the anisotropy saturation due to the kinetic instability feedback behave similarly (both qualitatively and quantitatively) to the collisional-MHD description. ${ }^{11}$

Brunetti \& Lazarian (2011) appealed to theoretical arguments about the decrease of the effective mean free path and related isotropization of the particle distribution to argue that the collisionless damping of compressible modes will be reduced in the ICM compared to the calculations in earlier papers (Brunetti \& Lazarian 2007). ${ }^{12}$ Our present calculations do not account for the collisionless damping of compressible motions, but similar to Brunetti \& Lazarian (2011), we may argue that this type of damping is not important, at least for the large-scale compressions.

\subsection{Implications of the Present Study}

The dynamics of the ICM plasmas is important for understanding most of the ICM physics, including the formation of galaxy clusters and their evolution. The relaxation that we discussed in this paper explains how clusters can have magnetic field generation, as well as turbulent cascade present there. We showed that for sufficiently high rates of isotropization arising from the interaction of particles with magnetic fluctuations induced by plasma instabilities, the collisionless plasma becomes effectively collisional and can be described by an ordinary MHD approach. This can serve as a justification for earlier MHD studies of the ICM dynamics and can motivate new ones.

In general, ICM studies face one major problem. The estimated Reynolds number for the ICM using the Coulomb cross sections is small ( $\sim 100$ or less), so that one may even question the existence of turbulence in galaxy clusters. This is the problem that we deal with in the present paper and argue that the Reynolds numbers in the ICM may be much larger than the naive estimates above. The difference comes from the dramatic decrease of the mean free path of the particles due to the interaction of ions with fluctuations induced by plasma instabilities. In other words, our study shows that the collisional MHD approach may correctly represent properties of turbulence in the intracluster plasma. In particular, it indicates that MHD turbulence theory may be applicable to a variety of collisionless

\footnotetext{
11 We note, as remarked before, that while in the case of the ICM plasma the anisotropy relaxation rate is expected to be much larger than the dynamical rates of turbulent motions by several orders of magnitude, in the case of the solar wind the relaxation rate is only about 10 times larger than the characteristic compression rates, so that in this case an instantaneous relaxation of the anisotropy is not always applicable (Meng et al. 2012a; Chandran et al. 2011).

12 This happened to be important for cosmic-ray acceleration by fast modes (see Yan \& Lazarian 2002, 2004, 2008) that takes place in the ICM.
} 
media. This is a big extension of the domain of applicability of the Goldreich \& Sridhar (1995) theory of Alfvénic turbulence.

\section{SUMMARY AND CONCLUSIONS}

The plasma in the ICM is formally weakly collisional. Indeed, as far as Coulomb collisions are involved, the mean free paths of particles are comparable to size of galaxy clusters as a result of the high temperatures and low densities of the intracluster plasmas. Therefore, one might expect the plasmas to have high viscosity and not allow turbulent motions. At the same time, magnetic fields and turbulence are observed to be present there. The partial resolution of the paradox may be that even small magnetic fields can substantially decrease the perpendicular viscosity of plasmas and enable Alfvénic turbulence that is weakly coupled with the compressible modes (see also Lazarian 2006b). Our present work indicates that the parallel viscosity of plasmas can also be reduced compared with the standard Braginskii values.

Aiming to understand the effects of the low collisionality on the turbulence statistics and on the turbulent magnetic field amplification in the ICM, both of which are commonly treated using a collisional-MHD description, we performed 3D numerical simulations of forced turbulence employing a singlefluid collisionless-MHD model. We focused on models with trans-sonic turbulence and at the high $\beta$ regime (where $\beta$ is the ratio between the thermal and magnetic pressures), which are conditions appropriate to the ICM. We also considered a model with low $\beta$ for comparison.

Our collisionless-MHD approach is based on the CGL-MHD model, the simplest fluid model for a collisionless plasma, which differs from the standard collisional-MHD by the presence of an anisotropic thermal pressure tensor. The new forces arising from this anisotropic pressure modify the MHD linear waves and produce the firehose and mirror instabilities. These instabilities in a macroscopic fluid can be viewed as the long-wavelength limit of the corresponding kinetic instabilities driven by the temperature anisotropy for which the higher the $\beta$ regime, the faster the growth rate.

Considering the feedback of the kinetic instabilities on the pressure anisotropy, we adopted a plausible model of anisotropy relaxation and modified the CGL-MHD equations in order to take into account the effects of relaxation of the anisotropy arising from the scattering of individual ions by fluctuations induced by plasma instabilities. This model appeals to earlier observational and numerical studies in the context of the solar magnetosphere, as well as theoretical considerations discussed in earlier works. While the details of this isotropization feedback are difficult to quantify from first principles, the rate at which an initial anisotropy is relaxed is found (at least in 2D PIC simulations) to be a few percent of the ion Larmor frequency (Gary et al. 1997, 1998, 2000). The frequencies that we deal with in our numerical simulations are much smaller than the ion Larmor frequency in the ICM (considering the scale of the computational domain $\sim 100 \mathrm{kpc}$ ). This has motivated us to consider this anisotropy relaxation to be instantaneous. Nevertheless, for completeness we also performed simulations with finite rates, in order to access their potential effects in the results.

The main results from our simulated models can be summarized as follows:

1. Anisotropy in the collisionless fluid is naturally created by turbulent motions as a consequence of fluctuations of the magnetic field and gas densities. In all our models, the net increase of magnetic field intensity led to the predominance of the perpendicular pressure in most of the volume of the domain.

2. In the high $\beta$ regime with moderate initial magnetic field, the model without anisotropy relaxation (which is therefore a "standard" CGL-MHD model; see Model A2 in Figures 1 and 2) has the PDF of the density broadened, especially in the low-density tail, in comparison to the collisionalMHD model. This is a consequence of the action of the mirror instability, which traps the gas in small cells of low magnetic field intensity. The density and velocity power spectra show excess of power especially at small scales, where the instabilities are stronger, although the magnetic field reveals less power. Consistently, the anisotropies in the structure functions of density, velocity, and magnetic field are reduced at the smallest scales in comparison to the collisional-MHD model.

3. Models with anisotropy relaxation (either instantaneous, or with the finite rates $10^{2}$ or $10^{3}$ times larger than the inverse of the turbulence turnover time $t_{\text {turb }}$ ) present density PDFs, power spectra, and anisotropy in structures that are very similar to the collisional MHD model. However, the model with the smallest anisotropy relaxation rate $\left(\sim 10 t_{\text {turb }}^{-1}\right)$ shows a little excess of power in density and velocity in the smallest scales, already in the dissipative range. This is consistent with the presence of instabilities in the smallest regions of the gas.

4. Models starting with a very weak, seed magnetic field (i.e., with very high $\beta$ ), without any anisotropy relaxation, have the magnetic energy saturated at levels many orders of magnitude smaller than kinetic energy. The value of the magnetic energy at this saturated state is shown to depend on the sonic Mach number of the turbulence; the smaller the sound speed, the higher this saturation value.

5. Models starting with a very weak, seed magnetic field, but with anisotropy relaxation (with instantaneous or finite rates), show an increase of the magnetic energy until values close to those achieved by the collisional-MHD model. The growth rate of the magnetic energy for the model with instantaneous relaxation rate is similar to the collisionalMHD model, but this rate is a little smaller for the models with a finite rate of the anisotropy relaxation, as one should expect.

6. In the low $\beta$ regime, the strength of the injected turbulence (trans-sonic and sub-Alfvénic) is not able to produce anisotropy fluctuations that trigger instabilities. The statistics of the turbulence is very similar to the collisional-MHD case, consistent with the fact that in this regime the pressure forces have minor importance.

All these results show that the applicability of the collisionalMHD approach for studying the dynamics of the ICM, especially in the turbulent dynamo amplification of the magnetic fields, is justified if the anisotropy relaxation rate provided by the kinetic instabilities is fast enough and the anisotropies are relaxed until the marginally stable values. As stressed before, the quantitative description of this process is still lacking, but if we assume that the results obtained for the anisotropy relaxation (usually studied in the context of the collisionless plasma of the solar wind) can be applied to the turbulent ICM, we should expect a relaxation rate much faster than the rates at which the anisotropies are created by the turbulence. 
Table 2

Space and Time Averages (Upper Lines) and Standard Deviations (Lower Lines) for the Models A That Have Moderate Initial Magnetic Fields $\left(\beta_{0}=200\right)$

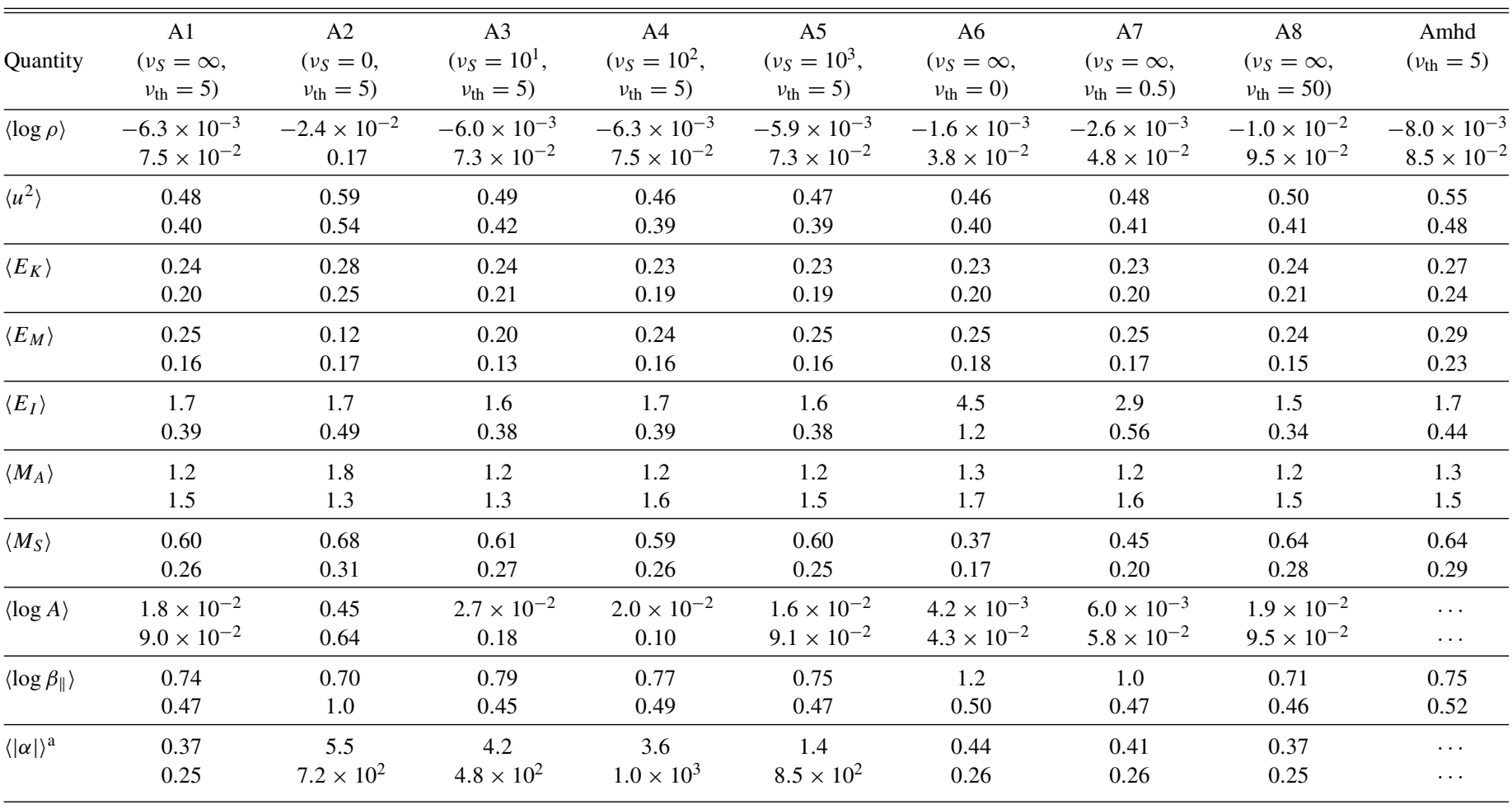

Note. ${ }^{\mathrm{a}} \alpha \equiv\left(p_{\|}-p_{\perp}\right) /\left(B^{2} / 4 \pi\right)$

We should emphasize that, even in the case of a good agreement between the collisional-MHD and collisionlessMHD results for the dynamics of the ICM, collisionless effects, like the kinetic instabilities themselves, can still be important for energetic processes in the ICM, such as the acceleration of particles (Kowal et al. 2011a, 2012), heating, and conduction (Narayan \& Medvedev 2001; Schekochihin et al. 2010; Kunz et al. 2011; Rosin et al. 2011; Riquelme et al. 2012).

R.S.L. acknowledges support from a grant of the Brazilian Agency FAPESP (2007/04551-0 and 2013/15115-8), EMGDP from FAPESP (2006/50654-3 and 2011/53275-4) and CNPq (306598/2009-4) grants. G.K. also acknowledges support from FAPESP (2009/50053-8, 2013/04073-2, and 2013/18815-0). D.F.G. thanks the European Research Council (ADG-2011 ECOGAL) and the Brazilian agencies CNPq (no. 300382/ 2008-1), CAPES (3400-13-1), and FAPESP (no.2011/129098) for financial support. A.L. acknowledges the NSF grant AST 1212096, the NSF-sponsored Center for Magnetic SelfOrganization, as well as the stimulating atmosphere of the International Institute of Physics (Natal, Brazil) and of the Harvard University during his sabbatical, and of the Vilas Associate Award. M.S.N. acknowledges support from FAPESP (2010/50298-8). The numerical simulations of this work were performed with the supercomputer of the Laboratory of Astroinformatics LAi (IAG/USP, NAT/Unicsul), whose purchase was made possible by FAPESP (grant 2009/54006-4).

\section{APPENDIX}

\section{SUPPLEMENTARY TABLES AND FIGURES}

Tables 2-4 present one-point statistics in space and time for the simulated models in Table 1 . The averaged quantities
Table 3

Space and Time Averages (Upper Lines) and Standard Deviations (Lower Lines) for Models B That Have Initial Strong Magnetic Field $(\beta=0.2)$

\begin{tabular}{lcc}
\hline \hline Quantity & B1 & Bmhd \\
\hline$\langle\log \rho\rangle$ & $-1.0 \times 10^{-2}$ & \\
\hline$\left\langle u^{2}\right\rangle$ & $9.8 \times 10^{-2}$ & $-1.8 \times 10^{-2}$ \\
& 0.90 & 0.12 \\
\hline$\left\langle E_{K}\right\rangle$ & 0.79 & 0.86 \\
& 0.44 & 0.73 \\
\hline$\left\langle E_{M}\right\rangle$ & 0.41 & 0.42 \\
& 4.8 & 0.39 \\
\hline$\left\langle E_{I}\right\rangle$ & 0.80 & 4.8 \\
& 1.7 & 0.90 \\
\hline$\left\langle M_{A}\right\rangle$ & 0.57 & 1.7 \\
& 0.28 & 0.75 \\
\hline$\left\langle M_{S}\right\rangle$ & 0.13 & 0.27 \\
& 0.83 & 0.13 \\
\hline$\langle\log A\rangle$ & 0.37 & 0.82 \\
& $5.5 \times 10^{-2}$ & 0.36 \\
\hline$\left\langle\log \beta_{\|}\right\rangle$ & 0.21 & $\ldots$ \\
\hline$\langle\alpha \mid\rangle^{\mathrm{a}}$ & -0.69 & $\cdots$ \\
\hline & 0.30 & -0.66 \\
\hline & $4.1 \times 10^{-2}$ & $\ldots .21$ \\
\hline & $3.9 \times 10^{-2}$ & $\ldots$ \\
\hline & &
\end{tabular}

Note. ${ }^{\mathrm{a}} \alpha \equiv\left(p_{\|}-p_{\perp}\right) /\left(B^{2} / 4 \pi\right)$. 

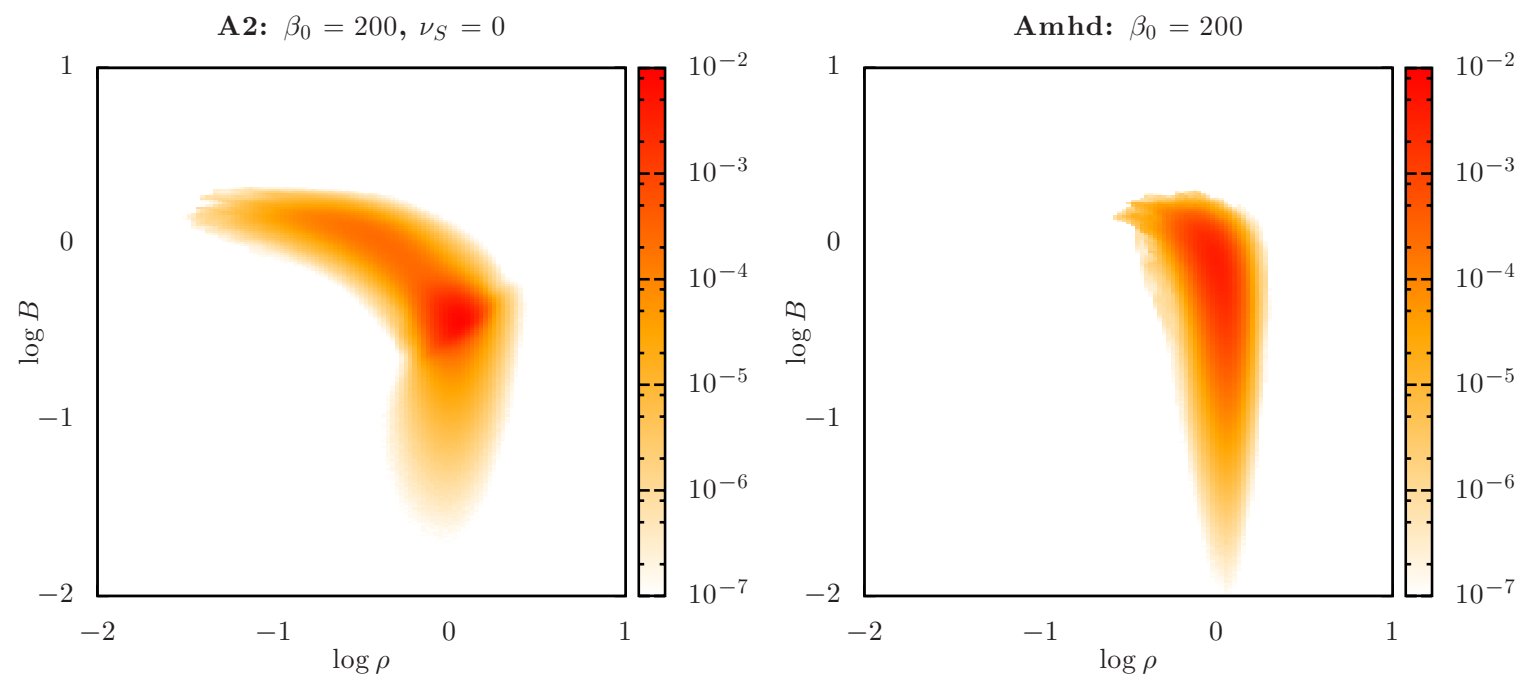

Figure 10. Two-dimensional normalized histograms of $\log \rho$ vs. $\log B$. Left: collisionless model A2 with null anisotropy relaxation rate. Right: collisional MHD model Amhd. The histograms were calculated using snapshots every $\Delta t=1$, from $t=2$ until the final time $t_{f}$ indicated in Table 1. See more details in Section 4.3 . (A color version of this figure is available in the online journal.)

Table 4

Space and Time Averages (Upper Lines) and Standard Deviations (Lower Lines) for Models C That Have Initial Very Weak (Seed) Magnetic Field

\begin{tabular}{lccccc}
\hline \hline Quantity & $\mathrm{C} 1$ & $\mathrm{C} 2$ & $\mathrm{C} 3$ & $\mathrm{C} 4$ & $\mathrm{Cmhd}$ \\
& $\left(v_{S}=\infty\right.$, & $\left(v_{S}=0\right.$, & $\left(v_{S}=0\right.$, & $\left(v_{S}=10^{2}\right.$, & $\left(V_{S 0}=1\right)$ \\
\hline$\langle\log \rho\rangle$ & $-8.5 \times 10^{-3}$ & $-1.5 \times 10^{-2}$ & $-8.7 \times 10^{-2}$ & $-8.8 \times 10^{-3}$ & $-9.1 \times 10^{-3}$ \\
& $8.7 \times 10^{-2}$ & 0.12 & 0.28 & $8.9 \times 10^{-2}$ & $9.0 \times 10^{-2}$ \\
\hline$\left\langle u^{2}\right\rangle$ & 0.79 & 0.70 & 0.79 & 0.80 & 0.79 \\
& 0.63 & 0.73 & 0.64 & 0.63 & 0.63 \\
\hline$\left\langle E_{K}\right\rangle$ & 0.38 & 0.32 & 0.37 & 0.38 & 0.38 \\
& 0.30 & 0.31 & 0.39 & 0.29 & 0.30 \\
\hline$\left\langle E_{M}\right\rangle$ & $6.2 \times 10^{-2}$ & $6.2 \times 10^{-6}$ & $1.0 \times 10^{-4}$ & $2.6 \times 10^{-2}$ & $9.0 \times 10^{-2}$ \\
& $7.5 \times 10^{-2}$ & $3.5 \times 10^{-5}$ & $3.7 \times 10^{-4}$ & $3.9 \times 10^{-2}$ & 0.11 \\
\hline$\left\langle E_{I}\right\rangle$ & 1.7 & 1.7 & 0.33 & 1.6 & 1.7 \\
& 0.49 & 0.44 & 0.28 & 0.49 & 0.51 \\
\hline$\left\langle M_{A}\right\rangle$ & 4.6 & $5.9 \times 10^{2}$ & $2.8 \times 10^{2}$ & 8.1 & 3.8 \\
& 6.7 & $5.7 \times 10^{2}$ & $4.2 \times 10^{2}$ & 11 & 5.3 \\
\hline$\left\langle M_{S}\right\rangle$ & 0.78 & 0.74 & 2.1 & 0.79 & 0.78 \\
& 0.34 & 0.38 & 1.0 & 0.34 & 0.34 \\
\hline$\langle\log A\rangle$ & $1.3 \times 10^{-2}$ & 0.51 & 2.1 & $6.7 \times 10^{-3}$ & $\ldots$ \\
& $3.2 \times 10^{-2}$ & 0.87 & 1.2 & $8.6 \times 10^{-2}$ & $\ldots$ \\
\hline$\left\langle\log \beta_{\|}\right\rangle$ & 1.5 & 5.5 & 2.0 & 2.0 & 1.4 \\
& 0.64 & 1.4 & 2.1 & 0.70 & 0.62 \\
\hline$\langle|\alpha|\rangle^{\mathrm{a}}$ & 0.52 & $8.8 \times 10^{5}$ & $5.5 \times 10^{4}$ & $1.5 \times 10^{2}$ & $\ldots$ \\
& 0.25 & $7.1 \times 10^{7}$ & $1.7 \times 10^{7}$ & $6.7 \times 10^{4}$ & $\ldots$ \\
\hline & & & &
\end{tabular}

Note. ${ }^{\mathrm{a}} \alpha \equiv\left(p_{\|}-p_{\perp}\right) /\left(B^{2} / 4 \pi\right)$.

are listed in the leftmost column. Each column presents the averages and below it the standard deviation for each model. For the statistics, we considered snapshots spaced in time by $\Delta t=1$, from $t=2$ (Tables 2 and 3) or $t_{f}-10$ (Table 4) until $t_{f}$ (the $t_{f}$ for each model is listed in Table 1). All the values are in code units and can be converted into physical units according to the prescription given in Section 3.2. The functional definitions (in terms of the code units) of the physical quantities listed are $E_{K}=\rho u^{2} / 2, E_{M}=B^{2} / 2, E_{I}=\left(p_{\perp}+p_{\|} / 2\right)$,
$M_{A}=u \rho^{1 / 2} / B, M_{S}=u(3 \rho)^{1 / 2} /\left(2 p_{\perp}+p_{\|}\right)^{1 / 2}$. For the collisional MHD models, the following definitions are used: $E_{I}=3 p / 2, M_{S}=u(\rho / p)^{1 / 2}, \beta_{\|}=\beta$.

Results shown in Figures 10-12 are complementary to the analysis presented in Section 4. Figure 10 compares the distribution density/magnetic field intensity between the models A2 and Amhd of Table 1. In Figures 11 and 12, the power spectrum of the compressible component of the velocity and of the magnetic field are compared to the power spectrum of the velocity field. 

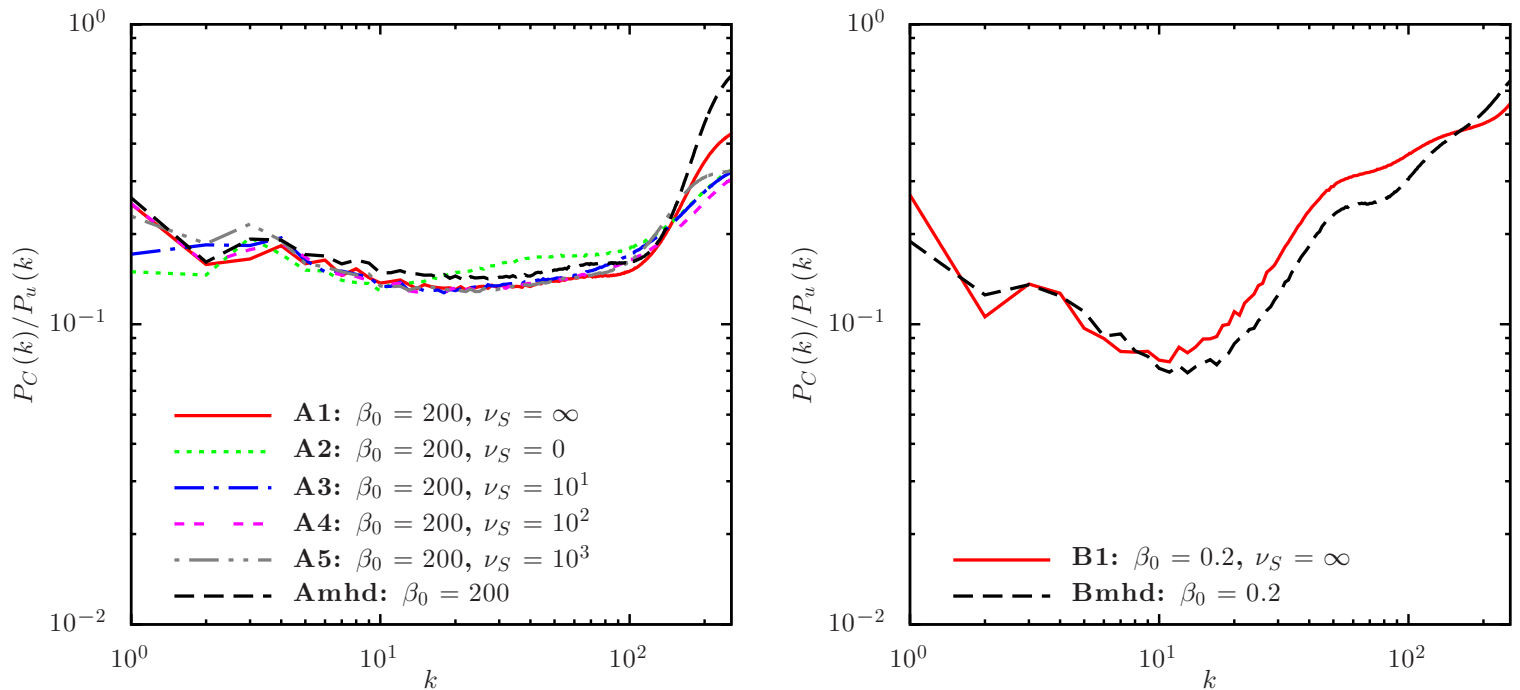

Figure 11. Ratio between the power spectrum of the compressible component $P_{C}(k)$ and the total velocity field $P_{u}(k)$, for the same models as in Figure 6 (see Table 1 and Section 4.4 for details).

(A color version of this figure is available in the online journal.)
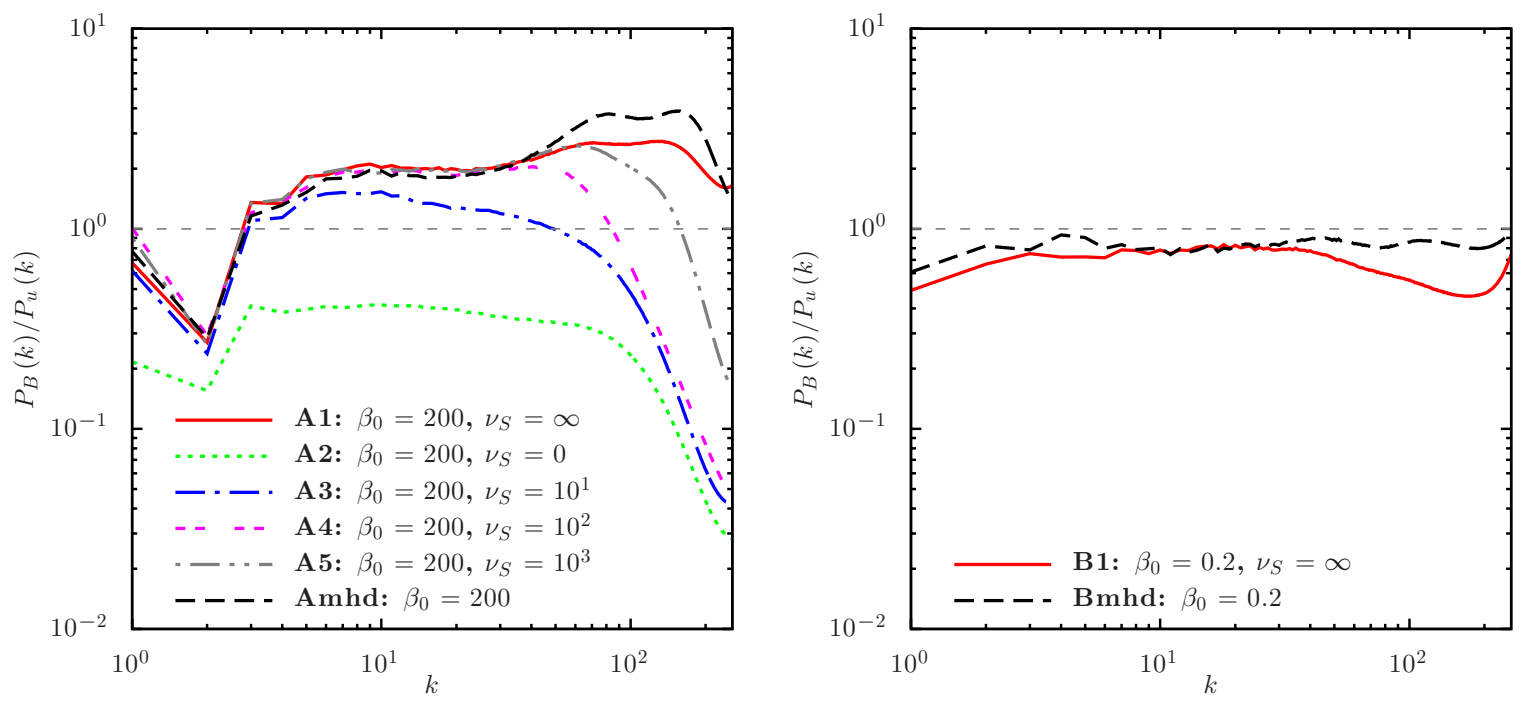

Figure 12. Ratio between the power spectrum of the magnetic field $P_{B}(k)$ and the velocity field $P_{u}(k)$ for the same models as in Figure 6 (see Table 1 and Section 4.4 for details).

(A color version of this figure is available in the online journal.)

Table 5

Power-law Fitting ${ }^{\mathrm{a}}$ for the Structure Function in the Direction Parallel ${ }^{\mathrm{b}}$ (Upper Lines) and Perpendicular ${ }^{\mathrm{c}}$ (Lower Lines) to the Local Mean Magnetic Field, for Different Models

\begin{tabular}{|c|c|c|c|c|c|c|c|c|}
\hline & $\begin{array}{c}\mathrm{A} 1 \\
\left(v_{S}=\infty\right. \\
\left.v_{\mathrm{th}}=5\right)\end{array}$ & $\begin{array}{c}\mathrm{A} 2 \\
\left(v_{S}=0\right. \\
\left.v_{\mathrm{th}}=5\right)\end{array}$ & $\begin{array}{c}\mathrm{A} 3 \\
\left(v_{S}=10^{1}\right. \\
\left.v_{\text {th }}=5\right)\end{array}$ & $\begin{array}{c}\mathrm{A} 4 \\
\left(v_{S}=10^{2}\right. \\
\left.v_{\text {th }}=5\right)\end{array}$ & $\begin{array}{c}\mathrm{A} 5 \\
\left(v_{S}=10^{3}\right. \\
\left.v_{\text {th }}=5\right)\end{array}$ & $\begin{array}{c}\text { Amhd } \\
\left(v_{\text {th }}=5\right)\end{array}$ & $\begin{array}{c}\mathrm{B} 1 \\
\left(v_{S}=\infty\right. \\
\left.v_{\mathrm{th}}=5\right)\end{array}$ & $\begin{array}{c}\text { Bmhd } \\
\left(v_{\text {th }}=5\right)\end{array}$ \\
\hline$\rho$ & $\begin{array}{l}0.93(0.03) \\
0.33(0.01)\end{array}$ & $\begin{array}{l}0.46(0.01) \\
0.18(0.00)\end{array}$ & $\begin{array}{l}0.89(0.02) \\
0.30(0.01)\end{array}$ & $\begin{array}{l}0.96(0.03) \\
0.36(0.01)\end{array}$ & $\begin{array}{l}0.95(0.03) \\
0.33(0.01)\end{array}$ & $\begin{array}{l}0.79(0.02) \\
0.29(0.01)\end{array}$ & $\begin{array}{l}1.04(0.01) \\
0.70(0.01)\end{array}$ & $\begin{array}{l}1.07(0.02) \\
0.63(0.01)\end{array}$ \\
\hline $\mathbf{u}$ & $\begin{array}{l}1.14(0.02) \\
0.74(0.01)\end{array}$ & $\begin{array}{l}0.86(0.01) \\
0.58(0.01)\end{array}$ & $\begin{array}{l}1.07(0.01) \\
0.73(0.01)\end{array}$ & $\begin{array}{l}1.12(0.02) \\
0.76(0.01)\end{array}$ & $\begin{array}{l}1.19(0.02) \\
0.76(0.01)\end{array}$ & $\begin{array}{l}1.05(0.01) \\
0.70(0.01)\end{array}$ & $\begin{array}{l}1.22(0.01) \\
0.64(0.01)\end{array}$ & $\begin{array}{l}1.26(0.01) \\
0.61(0.01)\end{array}$ \\
\hline B & $\begin{array}{l}0.70(0.02) \\
0.27(0.01)\end{array}$ & $\begin{array}{l}0.72(0.01) \\
0.35(0.01)\end{array}$ & $\begin{array}{l}0.72(0.02) \\
0.32(0.01)\end{array}$ & $\begin{array}{l}0.71(0.02) \\
0.30(0.01)\end{array}$ & $\begin{array}{l}0.72(0.02) \\
0.30(0.01)\end{array}$ & $\begin{array}{l}0.70(0.02) \\
0.26(0.01)\end{array}$ & $\begin{array}{l}1.22(0.02) \\
0.60(0.01)\end{array}$ & $\begin{array}{l}1.29(0.01) \\
0.60(0.01)\end{array}$ \\
\hline
\end{tabular}

Notes.

${ }^{a}$ A least-squares linear fitting was performed in the log-log plane. The errors are presented inside the parentheses. We considered $l_{\|, \perp}$ within the range of wavenumbers $[4,20]$ (approximately the inertial range inferred from the velocity power spectra, presented in Figure 6).

b $S_{2}\left(l_{\|}, 0\right) \propto l_{\|}^{\zeta}$.

c $S_{2}\left(0, l_{\perp}\right) \propto l_{\perp}^{\xi}$. 


\section{REFERENCES}

Bale, S. D., Kasper, J. C., Howes, G. G., et al. 2009, PhRvL, 103, 211101 Beresnyak, A. 2011, PhRvL, 106, 075001

Beresnyak, A. 2012a, PhRvL, 108, 035002

Beresnyak, A. 2012b, MNRAS, 422, 3495

Beresnyak, A., \& Lazarian, A. 2009, ApJ, 702, 1190

Beresnyak, A., \& Lazarian, A. 2010, ApJL, 722, L110

Beresnyak, A., \& Lazarian, A. 2013, in Magnetic Fields in Diffuse Media (submitted)

Brandenburg, A., Nordlund, A., Stein, R. F., \& Torkelsson, U. 1995, ApJ, 446, 741

Brandenburg, A., Sokoloff, D., \& Subramanian, K. 2012, SSRv, 169, 123

Brandenburg, A., \& Subramanian, K. 2005, PhR, 417, 1

Brunetti, G., \& Lazarian, A. 2007, MNRAS, 378, 245

Brunetti, G., \& Lazarian, A. 2011, MNRAS, 412, 817

Chandran, B. D. G., Dennis, T. J., Quataert, E., \& Bale, S. D. 2011, ApJ, 743, 197

Chew, G. F., Goldberger, M. L., \& Low, F. E. 1956, RSPSA, 236, 112

Cho, J., \& Lazarian, A. 2002, PhRvL, 88, 245001

Cho, J., \& Lazarian, A. 2003, MNRAS, 345, 325

Cho, J., Lazarian, A., \& Vishniac, E. T. 2002, ApJ, 564, 291

Cho, J., Vishniac, E. T., Beresnyak, A., Lazarian, A., \& Ryu, D. 2009, ApJ, 693, 1449

de Gouveia Dal Pino, E. M. 2006, in AIP Conf. Proc. 875, Plasma and Fusion Science: 16th IAEA Technical Meeting on Research using Small Fusion Devices (New York: AIP), 289

de Gouveia Dal Pino, E. M. 2011, in The Sun, the Stars, the Universe and General Relativity, ed. S. E. Perez Bergliaffa, M. Novello, \& R. Ruffini (Cambridge: Cambridge Scientific), 37

de Gouveia Dal Pino, E. M., Santos-Lima, R., Kowal, G., \& Falceta-Gonçalves, D. 2013, in IAU Symp. 294, Solar and Astrophysical Dynamos and Magnetic Activity, ed. A. Kosovichev (Cambridge: Cambridge Univ. Press), 337

de Lima, R. S., de Gouveia Dal Pino, E. M., Lazarian, A., \& Falceta-Gonçalves, D. 2009, in IAU Symp. 259, Cosmic Magnetic Fields: From Planets, to Stars and Galaxies, ed. K. G. Strassmeier, A. G. Kosovichev, \& J. E. Beckman (Cambridge: Cambridge Univ. Press), 563

Denton, R. E., Anderson, B. J., Gary, S. P., \& Fuselier, S. A. 1994, JGR, 99, 11225

Durret, F., \& Lima Neto, G. B. 2008, AdSpR, 42, 578

Enßlin, T., Vogt, C., \& Pfrommer, C. 2005, in The Magnetized Plasma in Galaxy Evolution (Krakow: Jagiellonian Univ.), 231

Gary, S. P. (ed.) 1993, Theory of Space Plasma Microinstabilities (Cambridge: Cambridge Univ. Press), 193

Gary, S. P., Li, H., O’Rourke, S., \& Winske, D. 1998, JGR, 103, 14567

Gary, S. P., Wang, J., Winske, D., \& Fuselier, S. A. 1997, JGR, 102, 27159

Gary, S. P., Yin, L., \& Winske, D. 2000, GeoRL, 27, 2457

Goldreich, P., \& Sridhar, S. 1995, ApJ, 438, 763

Govoni, F., \& Feretti, L. 2004, IJMPD, 13, 1549

Grasso, D., \& Rubinstein, H. R. 2001, PhR, 348, 163

Hall, A. N. 1979, JPIPh, 21, 431

Hall, A. N. 1980, MNRAS, 190, 371

Hall, A. N. 1981, MNRAS, 195, 685

Hau, L.-N., \& Wang, B.-J. 2007, NPGeo, 14, 557

Hazeltine, R. D., \& Mahajan, S. M. 2002, ApJ, 567, 1262

Hellinger, P., Trávníček, P., Kasper, J. C., \& Lazarus, A. J. 2006, GeoRL, 33, 9101

Keiter, P. A. 1999, PhD thesis, West Virginia Univ.

Kotarba, H., Lesch, H., Dolag, K., et al. 2011, MNRAS, 415, 3189

Kowal, G., de Gouveia Dal Pino, E. M., \& Lazarian, A. 2011a, ApJ, 735,102
Kowal, G., de Gouveia Dal Pino, E. M., \& Lazarian, A. 2012, PhRvL, 108,241102

Kowal, G., Falceta-Gonçalves, D. A., \& Lazarian, A. 2011b, NJPh, 13, 053001

Kowal, G., \& Lazarian, A. 2010, ApJ, 720, 742

Kulsrud, R. M. 1983, in Basic Plasma Physics: Selected Chapters, Handbook of Plasma Physics, Vol. 1, ed. A. A. Galeev \& R. N. Sudan (Amsterdam: North Holland), 1

Kulsrud, R. M. 2005, Plasma Physics for Astrophysics (Princeton Series in Astrophysics; Princeton, NJ: Princeton Univ. Press)

Kunz, M. W., Schekochihin, A. A., Cowley, S. C., Binney, J. J., \& Sanders, J. S. 2011, MNRAS, 410, 2446

Kuznetsov, V. D., \& Dzhalilov, N. S. 2010, PlPhR, 36, 788

Laganá, T. F., Lima Neto, G. B., Andrade-Santos, F., \& Cypriano, E. S. 2008, A\&A, 485, 633

Lazarian, A. 1992, A\&A, 264, 326

Lazarian, A. 2006a, AN, 327, 609

Lazarian, A. 2006b, ApJL, 645, L25

Lazarian, A., \& Beresnyak, A. 2006, MNRAS, 373, 1195

Le, A., Egedal, J., Fox, W., et al. 2010, PhPl, 17, 055703

Marsch, E. 2006, LRSP, 3, 1

Maruca, B. A., Kasper, J. C., \& Gary, S. P. 2012, ApJ, 748, 137

Meng, X., Tóth, G., Liemohn, M. W., Gombosi, T. I., \& Runov, A. 2012a, JGRA, 117,8216

Meng, X., Tóth, G., Sokolov, I. V., \& Gombosi, T. I. 2012b, JCoPh, 231, 3610

Narayan, R., \& Medvedev, M. V. 2001, ApJL, 562, L129

Nishimura, K., Gary, S. P., \& Li, H. 2002, JGRA, 107, 1375

Pudovkin, M. I., Besser, B. P., Lebedeva, V. V., Zaitseva, S. A., \& Meister, C.-V. 1999, PhPl, 6, 2887

Qu, H., Lin, Z., \& Chen, L. 2008, GeoRL, 35, 10108

Ramos, J. J. 2003, PhP1, 10, 3601

Riquelme, M. A., Quataert, E., Sharma, P., \& Spitkovsky, A. 2012, ApJ, 755,50

Rosin, M. S., Schekochihin, A. A., Rincon, F., \& Cowley, S. C. 2011, MNRAS, 413, 7

Samsonov, A. A., Alexandrova, O., Lacombe, C., Maksimovic, M., \& Gary, S. P. 2007, AnGeo, 25, 1157

Samsonov, A. A., \& Pudovkin, M. I. 2000, JGR, 105, 12859

Samsonov, A. A., Pudovkin, M. I., Gary, S. P., \& Hubert, D. 2001, JGR, 106,21689

Santos-Lima, R., de Gouveia Dal Pino, E. M., Lazarian, A., Kowal, G., \& Falceta-Gonçalves, D. 2011, in IAU Symp. 274, Advances in Plasma Astrophysics, ed. A. Bonanno, E. de Gouveia Dal Pino, \& A. G. Kosovichev (Cambridge: Cambridge Univ. Press), 482

Schekochihin, A. A., \& Cowley, S. C. 2006, PhPl, 13, 056501

Schekochihin, A. A., Cowley, S. C., Kulsrud, R. M., Hammett, G. W., \& Sharma, P. 2005, ApJ, 629, 139

Schekochihin, A. A., Cowley, S. C., Rincon, F., \& Rosin, M. S. 2010, MNRAS, 405, 291

Schekochihin, A. A., Cowley, S. C., Taylor, S. F., Maron, J. L., \& McWilliams, J. C. 2004, ApJ, 612, 276

Seough, J., \& Yoon, P. H. 2012, JGRA, 117, 8101

Sharma, P., Hammett, G. W., Quataert, E., \& Stone, J. M. 2006, ApJ, 637, 952

Snyder, P. B., Hammett, G. W., \& Dorland, W. 1997, PhPl, 4, 3974

Tajima, T., Mima, K., \& Dawson, J. M. 1977, PhRvL, 39, 201

Tanaka, M. 1993, JCoPh, 107, 124

Yan, H., \& Lazarian, A. 2002, PhRvL, 89, 1102

Yan, H., \& Lazarian, A. 2004, ApJ, 614, 757

Yan, H., \& Lazarian, A. 2008, ApJ, 673, 942

Yan, H., \& Lazarian, A. 2011, ApJ, 731, 35

Yoon, P. H., \& Seough, J. 2012, JGRA, 117, 8102

Zrake, J., \& MacFadyen, A. I. 2012, ApJ, 744, 32 